\title{
Infant formulae supplemented with prebiotics: Are they better than unsupplemented formulae? An updated systematic review
}

\author{
Agata Skórka, Małgorzata Pieścik-Lech, Maciej Kołodziej and Hania Szajewska* \\ Department of Paediatrics, The Medical University of Warsaw, Żwirki $i$ Wigury 63A, 02-091 Warsaw, Poland \\ (Submitted 9 August 2017 - Final revision received 27 December 2017 - Accepted 4 January 2018 - First published online 19 February 2018)
}

\begin{abstract}
In 2011, the Committee on Nutrition of the European Society for Paediatric Gastroenterology, Hepatology and Nutrition systematically reviewed published evidence related to the safety and health effects of the administration of formulae supplemented with pro- and/or prebiotics compared with unsupplemented formulae. We updated evidence on the effects of the administration of prebiotic-supplemented infant formulae (IF) compared with unsupplemented IF. Five databases were searched up to March 2017 for randomised controlled trials. In all, forty-one publications were identified, including twenty-five new publications. The administration of currently evaluated prebioticsupplemented formulae to healthy infants does not raise safety concerns with regard to growth and adverse effects. Some favourable clinical effects are possible, primarily stool softening, which may be beneficial in some infants. Currently, there is no existing robust evidence to recommend the routine use of prebiotic-supplemented formulae. The latter conclusion may reflect the small amount of data on specific prebiotics and outcomes, rather than a genuine lack of an effect. The efficacy and safety should be considered for each prebiotic(s)supplemented formula.
\end{abstract}

Key words: Infants: Nutrition: Microbiota: Feeding

In 2011, the Committee on Nutrition of the European Society for Paediatric Gastroenterology, Hepatology and Nutrition (ESPGHAN) systematically reviewed published evidence related to the safety and health effects of the administration of formulae supplemented with pro- and/or prebiotics compared with unsupplemented formulae ${ }^{(1)}$.

With regard to probiotics, in line with the 2011 ESPGHAN document $^{(1)}$, our recent updated systematic review ${ }^{(2)}$ concluded that the available scientific data suggest that the administration of currently evaluated probiotic-supplemented formulae to healthy infants does not raise safety concerns with regard to growth and adverse effects. Some beneficial clinical effects are possible; however, there is no existing robust evidence to recommend their routine use. The latter conclusion may reflect the small amount of data on a specific probiotic strain(s) and outcomes, rather than a genuine lack of an effect. The efficacy and safety should be considered for each probiotic(s)-supplemented formula.

With regard to prebiotic-supplemented formulae, in 2011 the ESPGHAN Committee concluded that the administration of currently evaluated prebiotic-supplemented formulae to healthy infants does not raise safety concerns with regard to growth and adverse effects. The Committee did not support the routine use of prebiotic-supplemented formulae in infants ${ }^{(1)}$. Subsequent to the Committee review, new evidence on the effects of supplementation of infant formulae with prebiotics was published. Here, we aimed to update the 2011 evidence on the effects of the administration of prebiotic-supplemented infant formulae to find out whether there is a need to revise current recommendations.

\section{Methods}

\section{Criteria for considering studies for this review}

The same methodology that has been already presented in two previous reviews ${ }^{(1,2)}$ was followed. In brief, only randomised controlled trials (RCT) were eligible for inclusion. Participants had to be healthy term infants. Only studies that compared use of infant formula or follow-on formula supplemented with prebiotics (with prebiotic specification) during the manufacturing process compared with unsupplemented formula were included. Studies in which prebiotics were not administered during the manufacturing process, but thereafter, for example in capsules, the contents of which were supplemented to infant formula or feeds, were excluded. Studies in which synthetic human milk oligosaccharides were used were excluded. We also excluded trials evaluating fermented, acidified, and partially or extensively

Abbreviations: AOS, acidic oligosaccharide; ESPGHAN, European Society for Paediatric Gastroenterology, Hepatology and Nutrition; FOS, long-chain fructooligosaccharide; GOS, galacto-oligosaccharides; MD, mean difference; PDX, polydextrose; RCT, randomised controlled trial; RR, risk ratio.

* Corresponding author: H. Szajewska, email hania@ipgate.pl 
hydrolysed formulae supplemented with prebiotics. We focused on growth and such clinical outcomes as gastrointestinal infections, respiratory infections, tolerance, etc. However, no firm definitions of any of these clinical outcomes were applied. Of note, in some trials, these outcomes were reported as the primary or secondary outcomes; in others, these were reported as adverse events. In this review, we evaluated them as referred to in the original publications.

\section{Search methods for identification of studies}

Five databases were searched up to March 2017 to identify potential studies: Cochrane Library, MEDLINE, EMBASE, Web of Science and CINAHL. No restrictions by either date or language were used in the search strategies. The search results were imported into Endnote bibliographic software, providing a total of 3035 records to be screened by the research team. The search strategy for one of the databases (EMBASE) is provided in the online Supplementary Table S1. We also searched reference lists of identified studies, key review articles and previous systematic reviews. Certain publication types (i.e. letters to the editor, abstracts, proceedings from scientific meetings) were excluded, unless a full set of data were obtained from the authors. Three registers for clinical trials (www.clinicaltrials.gov, www.clinical trialsregister.eu; www.anzctr.org.au) were screened.

\section{Data collection, analysis, extraction and management}

Titles and abstracts of the papers identified by the search strategy were independently screened by three researchers (A. S., M. K., M. P.-L.). Full texts of the potentially relevant publications were obtained and assessed. Any disagreements were discussed within the study team until a consensus was reached. Data extraction was performed using standard data-extraction forms. In addition to data such as methods, participants, interventions and outcomes, we collected information about sample size calculation and the funding of each study.

\section{Assessment of risk of bias in included studies}

The Cochrane Collaboration's tool for assessing risk of bias was used to establish the risk of bias. Type of randomisation method (selection bias), allocation concealment (selection bias), blinding of participants, personnel, intervention (performance bias), blinding of outcome assessment (detection bias), incomplete outcome data (attrition bias), selective reporting (reporting bias) and other forms of bias were considered ${ }^{(3)}$ (Fig. 1).

\section{Measures of treatment effect}

For reporting the effect, the results for individual studies and pooled statistics (if applicable) are reported as the risk ratio (RR), or mean difference (MD), between the experimental and control groups with $95 \% \mathrm{CI}$. In other circumstances, we report the findings as reported by the authors of the included studies.

\section{Data synthesis (statistical methods)}

If appropriate, the data were analysed using Review Manager (RevMan) (Computer program; version 5.3, 2014; The Cochrane
Collaboration). As various prebiotics differ in their effects, we did not perform pooling data (meta-analysis) of all prebiotic trials. Instead, we report evidence related to a specific prebiotic or their combinations separately. Compared with the 2011 report, no new meta-analyses were performed.

\section{Results \\ Description of studies}

For a flow diagram documenting the identification process for eligible trials (online Supplementary Fig. S1). Overall, forty-one publications met our inclusion criteria. In addition to the previously identified sixteen publications, twenty-five new publications were identified. In addition, four registered trials were identified: still recruiting at the time of the writing of this manuscript: NCT01143233, NCT03090360 and NCT02948114; active but not recruiting at the time of the writing of this manuscript: ACTRN12616001571460.

Table 1 summarises the characteristics of all included RCT evaluating the effects of prebiotic-supplemented formulae. Among new studies identified, there were three studies that were not included in the previous Committee systematic review despite having been published before the review ${ }^{(10,16,18)}$. A number of RCT described the same study population but reported different outcome measures ${ }^{(6,7,11,24,26,30,31,34-42)}$. The online Supplementary Table S2 summarises the characteristics of the excluded trials, with reasons for exclusion. All of the included studies were carried out in healthy term infants. The vast majority of trials were conducted in Westernised countries and the majority were industry-funded trials.

The studies varied in the types of prebiotics used. As previously reported by the ESPGHAN Committee on Nutrition, the most commonly studied prebiotic was a 9:1 mixture of short-chain galacto-oligosaccharides (GOS) and long-chain fructo-oligosaccharides (FOS) (GOS/FOS) ${ }^{(16-23,30,36-38,40,42,43)}$.

Other prebiotics studied were as follows:

(1) $\operatorname{GOS}^{(5-7,10,11,14,15,33,39)}$;

(2) acidic oligosaccharides (AOS) ${ }^{(4)}$;

(3) $\operatorname{FOS}^{(8,9,12,13,29,32)}$;

(4) GOS/FOS/AOS ${ }^{(4,24,31,34,35,44)}$;

(5) oligofructose plus inulin $(\mathrm{SYN}-1)^{(23,28)}$; and

(6) polydextrose (PDX) plus GOS with lactulose (PDX + GOS + LOS $)^{(25,27)}$ or without lactulose $(\mathrm{PDX}+\mathrm{GOS})^{(5,25-27,41)}$.

The doses of prebiotics ranged from $0 \cdot 1$ to $0 \cdot 8 \mathrm{~g} / 100 \mathrm{ml}$, and the duration of the intervention ranged from 2 weeks to 12 months. All but five $\operatorname{RCT}^{(9,10,14,31,34)}$ reported the prebiotic supplementation of an infant formula. In these five trials, prebiotics were used to supplement follow-on formula.

\section{Risk of bias in included trials}

The quality of the included RCT varied (Fig. 1). Almost all of the included trials had a number of methodological limitations. The most common problems were a lack of description of randomisation procedures and/or allocation concealment and/or blinding. 


\section{N British Journal of Nutrition}

Table 1. Characteristics of included randomised controlled trials in healthy term infants fed formulae supplemented with pro- and/or prebiotics compared with unsupplemented formulae (search up to March 2017). Overview of the findings: clinical results

(Mean values with their standard errors; medians amd 25-75 percentiles; risk ratios (RR), mean differences (MD) and $95 \%$ confidence intervals)

\begin{tabular}{|c|c|c|c|c|c|c|}
\hline References (country) & $\begin{array}{l}\text { Participants (age at } \\
\text { enrolment) }\end{array}$ & Intervention & Comparison & $\begin{array}{l}\text { Duration of } \\
\text { intervention } \\
\text { (follow-up) }\end{array}$ & Funding & $\begin{array}{l}\text { Effect (prebiotic-supplemented formula } \\
\text { group } v \text {. unsupplemented formula group) }\end{array}$ \\
\hline \multicolumn{7}{|l|}{ Growth } \\
\hline Fanaro et al. ${ }^{(4)}$ (Italy) ${ }^{*}$ & Healthy term infants & $\begin{array}{l}\text { GOS/FOS + AOS }(0.8 \mathrm{~g} / 100 \mathrm{ml})(\mathrm{n} 15) \\
\text { AOS }(0.2 \mathrm{~g} / 100 \mathrm{ml})(\mathrm{n} 16)\end{array}$ & $\mathrm{IF}(n 15)$ & 6 weeks & No info & NS \\
\hline Ashley et al. ${ }^{(5)}$ (USA) $\dagger$ & Healthy term infants & GOS $(0.4 \mathrm{~g} / 100 \mathrm{ml})(n 138)$ & IF $(n$ 142) & $120 \mathrm{~d}$ & Unclear & NS \\
\hline Ben et al. ${ }^{(6,7)}(\text { China })^{*}$ & Term infants & GOS $(0.24 \mathrm{~g} / 100 \mathrm{ml})(n 69)^{\prime}$ & $\begin{array}{l}\mathrm{IF}(n 52) \\
\mathrm{BF}(n 26)\end{array}$ & 6 months & No info & NS \\
\hline Bettler-Euler et al. ${ }^{(8)}(\mathrm{USA})^{\star}$ & Healthy term infants & $\begin{array}{l}\text { FOS }(0.3 \mathrm{~g} / 100 \mathrm{ml})(n 101) \\
\text { FOS }(0.15 \mathrm{~g} / 100 \mathrm{ml})(n 98)\end{array}$ & IF $(n 98)$ & 12 weeks & No info & NS \\
\hline Brunser et al. $^{(9)}(\text { Chile })^{*}$ & $\begin{array}{l}\text { Healthy term infants, } \\
\text { enrolled at } 3.5 \text { months }\end{array}$ & $\begin{array}{l}\text { FOS }(0.2 \mathrm{~g} / 100 \mathrm{ml})(n 32) \\
\mathrm{L} \text { johnsonii La1 } 10^{8} \mathrm{CFU} / \mathrm{g}(n 25)\end{array}$ & $\begin{array}{l}\mathrm{FF}(n 33) \\
\mathrm{BF}(n 26)\end{array}$ & $\begin{array}{l}13 \text { weeks } \\
\text { (15 weeks) }\end{array}$ & No info & NS \\
\hline Fanaro et al..$^{(10)}$ (Italy) $\dagger$ & Healthy infants 4-6 months & $\begin{array}{l}\text { GOS }(0.5 \mathrm{~g} / 100 \mathrm{ml})(n 77 \text { including } \\
15 \text { preterm) }\end{array}$ & $\begin{array}{l}\text { IF ( } n 82 \text { including } \\
13 \text { preterm }\end{array}$ & 18 weeks & $\begin{array}{l}\text { Humana } \mathrm{GmbH}, \\
\text { Herford, Germany }\end{array}$ & NS \\
\hline Giovannini et al. ${ }^{(11)}$ (Italy)† & $<15 d$ & $\operatorname{GOS}(0.4 \mathrm{~g} / 100 \mathrm{ml})(n 83)$ & $\begin{array}{l}\mathrm{IF}(n 80) \\
\mathrm{BF}(n 199)\end{array}$ & 6 months & No info & NS \\
\hline Paineau et al. ${ }^{(12)}$ (France)† & Healthy, term infants $<7 d$ & FOS $0.4 \mathrm{~g} / 100 \mathrm{ml}(n 31)$ & IF (n 27) & 4 months & No info & NS \\
\hline Ripoll et al. ${ }^{(13)}$ (Spain)† & $\begin{array}{l}\text { Healthy infants } \\
4 \text { months } \pm 2 \text { weeks }\end{array}$ & FOS $(0.5 \mathrm{~g} / \mathrm{ml})(n 38)$ & IF ( $n$ 37) & 6 months & $\begin{array}{l}\text { Syrallberia S.A.U. } \\
\text { Saragossa, Spain }\end{array}$ & NS \\
\hline Sierra et al. ${ }^{(14)}($ Spain)† & $\begin{array}{l}\text { Healthy term infants } \\
<2 \text { months }\end{array}$ & $\begin{array}{c}\text { GOS }(0.44 \mathrm{~g} / 100 \mathrm{ml} \text { in IF and } \\
0.50 \mathrm{~g} / \mathrm{dl} \text { in FF) }(n 188)\end{array}$ & $\mathrm{IF}(n 177)$ & 12 months & Hero Group & NS \\
\hline Williams et al. ${ }^{(15)}$ (USA)† & Healthy term $<8 d$ & $\begin{array}{l}\text { GOS }(0.4 \mathrm{~g} / 100 \mathrm{ml})(n 46) \\
\text { GOS }(0.8 \mathrm{~g} / 100 \mathrm{ml})(n 43)\end{array}$ & IF $(n 44)$ & $119 d$ & Abbott Nutrition & NS \\
\hline Bisceglia et al. ${ }^{(16)}$ (Italy)† & Healthy term infants & GOS/FOS $(0.8 \mathrm{~g} / 100 \mathrm{ml})(n 39)$ & IF ( $n$ 37) & $28 d$ & $\begin{array}{l}\text { Partially financed by } \\
\text { Numico } \\
\text { Friedrichsdorf } \\
\text { Germany }\end{array}$ & NS \\
\hline Costalos et al. ${ }^{(17)}(\text { Greece })^{\star}$ & $\begin{array}{l}\text { Healthy term infants, } \\
\text { enrolled }<14 \mathrm{~d}\end{array}$ & GOS/FOS $(0.4 \mathrm{~g} / 100 \mathrm{ml})(n 80)$ & $\mathrm{IF}(n 80)$ & 12 weeks & No info & NS \\
\hline Bruzzese et al. ${ }^{(18)}$ (Italy) ${ }^{*}$ & Healthy infants $15-120 \mathrm{~d}$ & $\underset{(n \text { GOS/FOS }}{\operatorname{G}(0.4 \mathrm{~g} / 100 \mathrm{ml})}$ & IF $(n$ 173) & 12 months & $\begin{array}{l}\text { An unrestricted grant } \\
\text { from } \\
\text { Numico Research, } \\
\text { Friedrichsdor, } \\
\text { Germany }\end{array}$ & $\begin{array}{l}\text { Increased mean body weight at } 3 \text { and } \\
6 \text { months of follow-up }(P<0.01) \text {. } \\
\text { Increased mean body length at all-time } \\
\text { intervals }(P<0.05)\end{array}$ \\
\hline Decsi et al. ${ }^{(19)}$ (Hungary)* $^{*}$ & Healthy term infants & GOS/FOS $(0.4 \mathrm{~g} / 100 \mathrm{ml})(n 14)$ & $\begin{array}{l}\mathrm{IF}(n 13) \\
\mathrm{BF}(n 42)\end{array}$ & 12 weeks & No info & NS \\
\hline $\begin{array}{l}\text { Ivakhnenko et al. }{ }^{(20)} \\
\text { (Ukraine)† }\end{array}$ & Newborns & GOS/FOS $(0.8 \mathrm{~g} / 100 \mathrm{ml})(n 80)$ & $\begin{array}{l}\mathrm{IF}(n 80) \\
\mathrm{BF}(n 80)\end{array}$ & $\begin{array}{l}2 \text { months (follow-up } \\
18 \text { months) }\end{array}$ & $\begin{array}{l}\text { Pediatrics } \\
\text { Department of Lviv } \\
\text { National Medical } \\
\text { University, } \\
\text { state registration } \\
\text { number, } \\
\text { 0108U101130 }\end{array}$ & NS \\
\hline Moro et al. ${ }^{(21)}$ (Italy) ${ }^{*}$ & Term infants & $\begin{array}{l}\text { GOS/FOS }(0.4 \mathrm{~g} / 100 \mathrm{ml})(n 30) \\
\text { GOS/FOS }(0.8 \mathrm{~g} / 100 \mathrm{ml})\end{array}$ & IF ( $n$ 33) & 4 weeks & No info & NS \\
\hline Salvini et al. ${ }^{(22)}$ (Italy) $\dagger$ & $\begin{array}{l}\text { Healthy newborns of } \\
\text { hepatitis C virus-infected } \\
\text { mothers }\end{array}$ & GOS/FOS $(0.8 \mathrm{~g} / 100 \mathrm{ml})(n 10)$ & $\mathrm{IF}(n 10)$ & 6 months & $\begin{array}{l}\text { Danone Research, } \\
\text { Centre for } \\
\text { Specialised } \\
\text { Nutrition }\end{array}$ & NS \\
\hline $\begin{array}{l}\text { Veereman-Wauters } \\
\text { et al. }{ }^{(23)} \text { (Belgium) } \dagger\end{array}$ & Healthy term infants $<5 d$ & $\begin{array}{l}\text { Oligofructose-enriched inulin } \\
\text { (SYN1) }(0.4 \mathrm{~g} / 100 \mathrm{ml})(n 21) \\
\text { Oligofructose-enriched inulin (SYN-1) } \\
(0.8 \mathrm{~g} / 100 \mathrm{ml})(n 20) \\
\text { GOS:FOS }(0.8 \mathrm{~g} / 100 \mathrm{ml})(n 19)\end{array}$ & $\begin{array}{l}\mathrm{IF}(n 21) \\
\mathrm{BF}(n 29)\end{array}$ & $28 d$ & Beneo-Orafti & NS \\
\hline
\end{tabular}




\begin{tabular}{|c|c|c|c|c|c|c|}
\hline References (country) & $\begin{array}{l}\text { Participants (age at } \\
\text { enrolment) }\end{array}$ & Intervention & Comparison & $\begin{array}{l}\text { Duration of } \\
\text { intervention } \\
\text { (follow-up) }\end{array}$ & Funding & $\begin{array}{l}\text { Effect (prebiotic-supplemented formula } \\
\text { group } v \text {. unsupplemented formula group) }\end{array}$ \\
\hline $\begin{array}{l}\text { Piemontese et al. } \text { (24) }^{\text {(Italy, }} \\
\text { Switzerland, Germany, } \\
\text { Austria, the Netherlands) } \\
\text { (The same population as } \\
\text { van Stuijvenberg) } \dagger\end{array}$ & $\begin{array}{l}\text { Healthy, term infants } \\
<8 \text { weeks }\end{array}$ & $\underset{(n \text { 4 } 14)}{\mathrm{GOS} / \mathrm{FOS}}+\mathrm{AOS}(0 \cdot 8 \mathrm{~g} / 100 \mathrm{ml})$ & $\begin{array}{l}\mathrm{IF}(n 416) \\
\mathrm{BF}(n 300)\end{array}$ & 1 years & No info & NS \\
\hline Nakamura et al. ${ }^{(25)}(\mathrm{USA})^{*}$ & $\begin{array}{l}\text { Healthy term infants } 13 \\
\text { to } 92 \mathrm{~d}\end{array}$ & $\begin{array}{l}\mathrm{PDX}+\mathrm{GOS}(0.4 \mathrm{~g} / 100 \mathrm{ml})(n 27) \\
\mathrm{PDX}+\mathrm{GOS}+\mathrm{LOS} 0.4 \mathrm{~g} / 100 \mathrm{ml} \\
(n 27) \\
\mathrm{PDX}+\mathrm{GOS}+\mathrm{LOS} 0.8 \mathrm{~g} / 100 \mathrm{ml} \\
(n 25)\end{array}$ & $\begin{array}{l}\mathrm{IF}(n 25) \\
\mathrm{BF}(n 30)\end{array}$ & $28 d$ & $\begin{array}{l}\text { Mead Johnson \& } \\
\text { Company, } \\
\text { Evansville, IN }\end{array}$ & NS \\
\hline Scalabrin et al. ${ }^{(26)}(\mathrm{USA}) \dagger$ & $\begin{array}{l}\text { Healthy term infants } \\
21-30 \mathrm{~d}\end{array}$ & PDX/GOS $(0.4 \mathrm{~g} / 100 \mathrm{ml})(n 100)$ & $\begin{array}{l}\mathrm{IF}(n 101) \\
\mathrm{BF}(n 88)\end{array}$ & $60 d$ & $\begin{array}{l}\text { Mead Johnson } \\
\text { Nutrition }\end{array}$ & NS \\
\hline Ziegler et al..$^{(27)}(\text { USA })^{*}$ & Healthy term infants $<14 \mathrm{~d}$ & $\begin{array}{l}\text { PDX + GOS }(0.4 \mathrm{~g} / 100 \mathrm{ml})(n 74) \\
\text { PDX + GOS + LOS }(0.8 \mathrm{~g} / 100 \mathrm{ml}) \\
(n 76)\end{array}$ & IF $(n 76)$ & $120 \mathrm{~d}$ & Mead Johnson \& Co & NS \\
\hline $\begin{array}{l}\text { Closa-Monasterolo } \\
\text { et al. }\end{array}$ & Newborns & $\begin{array}{l}\text { Oligofructose-enriched inulin (SYN1) } \\
\qquad(0.8 \mathrm{~g} / 100 \mathrm{ml})(n \text { 128) }\end{array}$ & $\begin{array}{l}\mathrm{IF}(n 124) \\
\mathrm{BF}(n 136)\end{array}$ & 4 months & $\begin{array}{l}\text { BENEO Institute (an } \\
\text { Initiative of BENEO } \\
\text { GmbH). }\end{array}$ & NS \\
\hline $\begin{array}{l}\text { Tolerance } \\
\text { Fanaro et al. }{ }^{(4)} \text { (Italy) }{ }^{*}\end{array}$ & Healthy term infants & $\begin{array}{l}\text { GOS/FOS + AOS }(0.8 \mathrm{~g} / 100 \mathrm{ml})(n 15) \\
\text { AOS }(0.2 \mathrm{~g} / 100 \mathrm{ml})(n 16)\end{array}$ & $\mathrm{IF}(n 15)$ & 6 weeks & No info & $\begin{array}{l}\text { NS (crying, regurgitation, vomiting } \\
\text { episodes) }\end{array}$ \\
\hline $\begin{array}{l}\text { Paineau et al }{ }^{(12)} \text { (France) } \dagger \\
\text { Ripoll et al. }^{\left({ }^{13)}\right.} \text { (Spain)† }\end{array}$ & $\begin{array}{l}\text { Healthy, term infants }<7 d \\
\text { Healthy infants } \\
4 \text { months } \pm 2 \text { weeks }\end{array}$ & $\begin{array}{l}\text { FOS } 0.4 \mathrm{~g} / 100 \mathrm{ml}(n 31) \\
\text { FOS }(0.5 \mathrm{~g} / \mathrm{ml})(n 38)\end{array}$ & $\begin{array}{l}\text { IF }(n \text { 27) } \\
\text { IF }(n \text { 37) }\end{array}$ & $\begin{array}{l}4 \text { months } \\
6 \text { months }\end{array}$ & $\begin{array}{l}\text { No info } \\
\text { Syrallberia S.A.U. } \\
\text { Saragossa, Spain }\end{array}$ & $\begin{array}{l}\text { NS (abdominal pain, crying, nausea) } \\
\text { NS (constipation, crying, regurgitation) } \\
\text { Number of days with vomiting was lower } \\
(P=0.05) \text { in the FOS group }\end{array}$ \\
\hline Xia et al. ${ }^{(29)}(\mathrm{USA}) \dagger$ & Healthy term infants $<7 \mathrm{~d}$ & $\begin{array}{l}\text { FOS }(0.2 \mathrm{~g} / 100 \mathrm{ml})(n 25) \\
\text { FOS }(0.3 \mathrm{~g} / 100 \mathrm{ml})(n 26)\end{array}$ & $\begin{array}{l}\mathrm{IF}(n 24) \\
\mathrm{BF}(n 22)\end{array}$ & 4 weeks & Abbott Nutrition & NS (spitting up or vomiting) \\
\hline Ashley et al. ${ }^{(5)}(\mathrm{USA}) \dagger$ & Healthy term infants & $\begin{array}{l}\text { GOS }(0.4 \mathrm{~g} / 100 \mathrm{ml})(n 138) \\
\text { PDX/GOS }(0.4 \mathrm{~g} / 100 \mathrm{ml})\end{array}$ & $\mathrm{IF}(n$ 142) & $120 d$ & Unclear & NS (fussiness, gassiness) \\
\hline Ben et al. ${ }^{(6,7)}(\text { China })^{*}$ & Term infants & GOS $(0.24 \mathrm{~g} / 100 \mathrm{ml})(n 69)$ & $\begin{array}{l}\mathrm{IF}(n 52) \\
\mathrm{BF}(n 26)\end{array}$ & 6 months & No info & NS (crying, regurgitation, vomiting) \\
\hline Fanaro et al. ${ }^{(10)}$ (Italy)† & Healthy infants $4-6$ months & $\begin{array}{l}\text { GOS }(0.5 \mathrm{~g} / 100 \mathrm{ml})(n 77 \text { including } \\
15 \text { preterm) }\end{array}$ & $\begin{array}{l}\text { IF }(n 82 \text { including } \\
13 \text { preterm }\end{array}$ & 18 weeks & $\begin{array}{l}\text { Humana GmbH, } \\
\text { Herford, Germany }\end{array}$ & $\begin{array}{l}\text { NS (crying, regurgitation, vomiting, } \\
\text { flatulence) }\end{array}$ \\
\hline Bruzzese et al. ${ }^{(18)}{\text { (Italy })^{*}}$ & Healthy infants $15-120 \mathrm{~d}$ & $\underset{(n \text { 169) }}{\text { GOS/FOS }}(0 \cdot 4 \mathrm{~g} / 100 \mathrm{ml})$ & $\mathrm{IF}(n$ 173) & 12 months & $\begin{array}{l}\text { An unrestricted grant } \\
\text { from } \\
\text { Numico Research, } \\
\text { Friedrichsdorf, } \\
\text { Germany }\end{array}$ & NS (no data) \\
\hline Decsi et al. ${ }^{(19)}$ (Hungary) $)^{*}$ & Healthy term infants & GOS/FOS $(0.4 \mathrm{~g} / 100 \mathrm{ml})(n 14)$ & $\begin{array}{l}\mathrm{IF}(n 13) \\
\mathrm{BF}(n 42)\end{array}$ & 12 weeks & No info & $\begin{array}{l}\text { NS (excessive irritability, vomiting, } \\
\text { regurgitation, atopy) }\end{array}$ \\
\hline Moro et al. ${ }^{(21)}(\text { Italy })^{*}$ & Term infants & $\begin{array}{l}\text { GOS/FOS }(0.4 \mathrm{~g} / 100 \mathrm{ml})(n 30) \\
\text { GOS/FOS }(0.8 \mathrm{~g} / 100 \mathrm{ml}) \\
(n 27)\end{array}$ & $\mathrm{IF}(n 33)$ & 4 weeks & No info & NS (crying, regurgitation, vomiting) \\
\hline Costalos et al. ${ }^{(17)}(\text { Greece })^{*}$ & $\begin{array}{l}\text { Healthy term infants, } \\
\text { enrolled } \leq 14 \mathrm{~d}\end{array}$ & GOS/FÓS $(0.4 \mathrm{~g} / 100 \mathrm{ml})(n 80)$ & IF $(n 80)$ & 12 weeks & No info & NS (tolerance posseting) \\
\hline $\begin{array}{l}\text { Knol et al. }{ }^{(30)} \text { (Germany) } \\
\text { (The same study as } \\
\text { Haarman } 2005 \\
\text { (- different outcomes) }^{\star}\end{array}$ & $\begin{array}{l}\text { Fully formula-fed infants } \\
\quad 4-8 \text { weeks }\end{array}$ & GOS/FOS $(0.8 \mathrm{~g} / 100 \mathrm{ml})(n 24)$ & $\begin{array}{l}\mathrm{IF}(n 23) \\
\mathrm{BF}(n 21)\end{array}$ & 6 weeks & Numico Research & NS (flatulence, posseting) \\
\hline $\begin{array}{l}\text { Veereman-Wauters } \\
\text { et al. } \\
\text { (23) } \\
\text { (Belgium) } \dagger\end{array}$ & Healthy term infants $<5 d$ & GOS:FOS $(0.8 \mathrm{~g} / 100 \mathrm{ml})(n 19)$ & $\begin{array}{l}\mathrm{IF}(n 21) \\
\mathrm{BF}(n 29)\end{array}$ & $28 d$ & Beneo-Orafti & NS (regurgitation and vomiting scores) \\
\hline $\begin{array}{l}\text { Piemontese et al. }{ }^{(24)} \text { (Italy, } \\
\text { Switzerland, Germany, } \\
\text { Austria, the Netherlands) } \\
\text { (The same population as } \\
\text { van Stuijvenberg) } \dagger\end{array}$ & $\begin{array}{l}\text { Healthy, term infants } \\
<8 \text { weeks }\end{array}$ & $\underset{(n \text { 414) }}{\mathrm{GOS} / \mathrm{FOS}+\mathrm{AOS}(0.8 \mathrm{~g} / 100 \mathrm{ml})}$ & $\begin{array}{l}\mathrm{IF}(n 416) \\
\mathrm{BF}(n 300)\end{array}$ & 1 year & No info & $\begin{array}{l}\text { NS (spitting, posseting, vomiting, colic, } \\
\text { flatulence, cramps) }\end{array}$ \\
\hline
\end{tabular}




\begin{tabular}{|c|c|c|c|c|c|c|}
\hline References (country) & $\begin{array}{l}\text { Participants (age at } \\
\text { enrolment) }\end{array}$ & Intervention & Comparison & $\begin{array}{l}\text { Duration of } \\
\text { intervention } \\
\text { (follow-up) }\end{array}$ & Funding & $\begin{array}{l}\text { Effect (prebiotic-supplemented formula } \\
\text { group } v \text {. unsupplemented formula group) }\end{array}$ \\
\hline $\begin{array}{l}\text { van Stuijvenberg et al. }{ }^{(31)} \\
\text { (The Netherlands, } \\
\text { Austria, Switzerland, } \\
\text { Italy, Germany) } \dagger\end{array}$ & Healthy infants $<8$ weeks & $\begin{array}{l}\text { GOS/FOS + AOS }(0 \cdot 8 \mathrm{~g} / 100 \mathrm{ml}) \\
(n \text { 414) }\end{array}$ & $\begin{array}{l}\mathrm{IF}(n 416) \\
\mathrm{BF}(n 300)\end{array}$ & 1 year & Danone Research & $\begin{array}{l}\text { NS (duration of vomiting, number of } \\
\text { vomiting episodes) }\end{array}$ \\
\hline $\begin{array}{l}\text { Veereman-Wauters } \\
\text { et al. }{ }^{(23)} \text { (Belgium) } \dagger\end{array}$ & Healthy term infants $<5 d$ & $\begin{array}{l}\text { Oligofructose-enriched inulin SYN1 } \\
(0.4 \text { or } 0.8 \mathrm{~g} / 100 \mathrm{ml})\end{array}$ & $\begin{array}{l}\mathrm{IF}(n 21) \\
\mathrm{BF}(n 29)\end{array}$ & $28 d$ & Beneo-Orafti & $\begin{array}{l}\text { NS (regurgitation and vomiting scores) } \\
\text { no data }\end{array}$ \\
\hline Nakamura et al. ${ }^{(25)}(\mathrm{USA})^{\star}$ & $\begin{array}{l}\text { Healthy term infants } 13 \text { to } \\
92 \mathrm{~d}\end{array}$ & PDX/GOS $(0.4 \mathrm{~g} / 100 \mathrm{ml})$ & $\begin{array}{l}\mathrm{IF}(n 25) \\
\mathrm{BF}(n 30)\end{array}$ & $28 d$ & $\begin{array}{l}\text { Mead Johnson \& } \\
\text { Company, } \\
\text { Evansville, IN. }\end{array}$ & NS (fussiness, gassiness) \\
\hline Scalabrin et al. ${ }^{(26)}(\mathrm{USA}) \dagger$ & $\begin{array}{l}\text { Healthy term infants } \\
21-30 \mathrm{~d}\end{array}$ & PDX/GOS $(0.4 \mathrm{~g} / 100 \mathrm{ml})(n 100)$ & $\begin{array}{l}\mathrm{IF}(n \text { 101) } \\
\mathrm{BF}(n 88)\end{array}$ & $60 d$ & $\begin{array}{l}\text { Evansville, IN. } \\
\text { Mead Johnson } \\
\text { Nutrition }\end{array}$ & NS (fussiness, gassiness) \\
\hline $\begin{array}{l}\text { Stool frequency } \\
\text { Fanaro et al. }^{(4)} \text { (Italy) }{ }^{*}\end{array}$ & Healthy term infants & AOS $(0.2 \mathrm{~g} / 100 \mathrm{ml})(n 16)$ & $\mathrm{IF}(n 15)$ & 6 weeks & No info & NS \\
\hline $\begin{array}{l}\text { Wernimont et al. }{ }^{(32)} \\
\text { (USA) }\end{array}$ & Healthy term infants $5-15 d$ & FOS $(0.3 \mathrm{~g} / 100 \mathrm{ml})(n$ 47) & $\begin{array}{l}\mathrm{IF}(n 48) \\
\mathrm{BF}(n 50)\end{array}$ & 8 weeks & Wyeth Nutrition & NS \\
\hline Xia et al. ${ }^{(29)}$ (USA)† & Healthy term infants $<7 d$ & $\begin{array}{l}\text { FOS }(0.2 \mathrm{~g} / 100 \mathrm{ml})(n 25) \\
\text { FOS }(0.3 \mathrm{~g} / 100 \mathrm{ml})(n 26)\end{array}$ & $\begin{array}{l}\mathrm{IF}(n 24) \\
\mathrm{BF}(n 22)\end{array}$ & 4 weeks & Abbott Nutrition & NS \\
\hline Ashley et al. ${ }^{(5)}(\mathrm{USA}) \dagger$ & Healthy term infants & GOS $(0.4 \mathrm{~g} / 100 \mathrm{ml})(n 138)$ & IF $(n 142)$ & $120 d$ & Unclear & $\begin{array}{l}\text { mean stool frequency higher } \\
\text { week } 1 \text { (3.9 (SE 0.2) v. } 2.2 \text { (SE 0.2); } \\
P<0.05) \\
\text { week } 2(3.7 \text { (SE } 0.2) \text { v. } 2.2 \text { (SE } 0.2 \text { ); } \\
P<0.05) \text {. } \\
\text { This pattern continued through } 60 \mathrm{~d} \text { of } \\
\text { age; by } 90 \mathrm{~d} \text { of age }-\mathrm{NS}\end{array}$ \\
\hline Ben et al. ${ }^{(6,7)}(\text { China })^{*}$ & Term infants & GOS $(0.24 \mathrm{~g} / 100 \mathrm{ml})(n 69)$ & $\begin{array}{l}\mathrm{IF}(n 52) \\
\mathrm{BF}(n 26)\end{array}$ & 6 months & No info & Increased stool frequency (no data) \\
\hline Fanaro et al. ${ }^{(10)}$ (Italy) $\dagger$ & Healthy infants $4-6$ months & $\begin{array}{l}\text { GOS }(0.5 \mathrm{~g} / 100 \mathrm{ml})(n 77 \text { including } \\
15 \text { preterm) }\end{array}$ & $\begin{array}{l}\text { IF }(n 82 \text { including } \\
13 \text { preterm }\end{array}$ & 18 weeks & $\begin{array}{l}\text { Humana GmbH, } \\
\text { Herford, Germany }\end{array}$ & NS \\
\hline Matsuki et al. ${ }^{(33)}$ (Japan)† & $\begin{array}{l}\text { Healthy term infants } \\
31-54 \mathrm{~d}\end{array}$ & GOS $(0.3 \mathrm{~g} / 100 \mathrm{ml})(n 17)$ & $\mathrm{IF}(n 18)$ & 2 weeks & $\begin{array}{l}\text { Yakult Central } \\
\text { Institute }\end{array}$ & NS \\
\hline Sierra et al. ${ }^{(14)}$ (Spain)† & $\begin{array}{l}\text { Healthy term infants } \\
<2 \text { months }\end{array}$ & $\begin{array}{l}\text { GOS }(0.44 \mathrm{~g} / 100 \mathrm{ml} \text { in IF and } 0.50 \mathrm{~g} / \mathrm{dl} \\
\text { in FF) }(n 188)\end{array}$ & IF $(n 177)$ & 12 months & Hero Group & $\begin{array}{l}\text { At } 3 \text { months: } 1.45 \text { (SE } 0.97) \text { v. } 1.26 \\
\quad \text { (SE } 0.83 \text { ); } P<0.05) ; \\
\text { At } 4 \text { months: }(1.5 \text { (SE } 0.99) \text { v. } 1.26 \\
\quad \text { (SE } 0.94) ; P<0.05) ; \\
\text { At } 6,9 \text { and } 12 \text { months - NS }\end{array}$ \\
\hline Williams et al. ${ }^{(15)}$ (USA)† & Healthy term $<8 d$ & $\begin{array}{l}\text { GOS }(0.4 \mathrm{~g} / 100 \mathrm{ml})(n 46) \\
\text { GOS }(0.8 \mathrm{~g} / 100 \mathrm{ml})(n 43)\end{array}$ & IF $(n$ 44) & $119 \mathrm{~d}$ & Abbott Nutrition & NS \\
\hline Bisceglia et al. ${ }^{(16)}$ (Italy) $\dagger$ & Healthy term infants & GOS/FOS $(0.8 \mathrm{~g} / 100 \mathrm{ml})(n 39)$ & $\mathrm{IF}(n 7)$ & $28 d$ & $\begin{array}{l}\text { Partially financed by } \\
\text { Numico } \\
\text { Friedrichsdorf } \\
\text { Germany }\end{array}$ & $\begin{array}{l}\text { At } 28 \text { d: } 3.4 \text { (SE } 0.7 \text { ) v. } 1.7 \text { (SE 0.9); } \\
\quad P<0.001\end{array}$ \\
\hline Costalos et al. ${ }^{(17)}(\text { Greece })^{\star}$ & $\begin{array}{l}\text { Healthy term infants, } \\
\text { enrolled } \leq 14 \mathrm{~d}\end{array}$ & GOS/FOS $(0.4 \mathrm{~g} / 100 \mathrm{ml})(n 80)$ & IF $(n 80)$ & 12 weeks & No info & $\begin{array}{l}1.9 \text { (range: } 1.2-2.1) \text { v. } 1.6(1.1-1.9) \\
P=0.031\end{array}$ \\
\hline $\begin{array}{l}\text { Knol et al. }{ }^{\left({ }^{0}\right)} \text { (Germany) } \\
\text { (The same study as } \\
\text { Haarman } 2005 \\
\text { (- different outcomes) }^{*}\end{array}$ & $\begin{array}{l}\text { Fully formula-fed infants } \\
4-8 \text { weeks }\end{array}$ & GOS/FOS $(0.8 \mathrm{~g} / 100 \mathrm{ml})(n 24)$ & $\begin{array}{l}\mathrm{IF}(n 23) \\
\mathrm{BF}(n 21)\end{array}$ & 6 weeks & Numico Research & NS \\
\hline Moro et al. ${ }^{(21)}(\text { Italy })^{*}$ & Term infants & $\begin{array}{l}\text { GOS/FOS }(0.4 \mathrm{~g} / 100 \mathrm{ml})(n 30) \\
\text { GOS/FOS }(0.8 \mathrm{~g} / 100 \mathrm{ml}) \\
(n 27)\end{array}$ & $\mathrm{IF}(n 33)$ & 4 weeks & No info & NS \\
\hline $\begin{array}{l}\text { Veereman-Wauters } \\
\text { et al. }{ }^{(23)} \text { (Belgium) } \dagger\end{array}$ & Healthy term infants $<5 d$ & $\begin{array}{l}\text { GOS/FOS }(0.8 \mathrm{~g} / 100 \mathrm{ml}) \\
\text { Oligofructose-enriched inulin SYN1 } \\
(0.4 \text { or } 0.8 \mathrm{~g} / 100 \mathrm{ml})\end{array}$ & $\begin{array}{l}\mathrm{IF}(n 21) \\
\mathrm{BF}(n 29)\end{array}$ & $28 d$ & Beneo-Orafti & NS \\
\hline
\end{tabular}




\begin{tabular}{|c|c|c|c|c|c|c|}
\hline References (country) & $\begin{array}{l}\text { Participants (age at } \\
\text { enrolment) }\end{array}$ & Intervention & Comparison & $\begin{array}{l}\text { Duration of } \\
\text { intervention } \\
\text { (follow-up) }\end{array}$ & Funding & $\begin{array}{l}\text { Effect (prebiotic-supplemented formula } \\
\text { group } v \text {. unsupplemented formula group) }\end{array}$ \\
\hline Fanaro et al. ${ }^{(4)}$ (Italy) ${ }^{*}$ & Healthy term infants & GOS/FOS + AOS $(0.8 \mathrm{~g} / 100 \mathrm{ml})(n 15)$ & $\mathrm{IF}(n$ 15) & 6 weeks & No info & Increased stool frequency (no exact data) \\
\hline $\begin{array}{l}\text { Piemontese et al. }{ }^{(24)} \text { (Italy, } \\
\text { Switzerland, Germany, } \\
\text { Austria, the Netherlands) } \\
\text { (The same population as } \\
\text { van Stuijvenberg) } \dagger\end{array}$ & $\begin{array}{l}\text { Healthy, term infants } \\
<8 \text { weeks }\end{array}$ & $\begin{array}{l}\text { GOS/FOS + AOS }(0.8 \mathrm{~g} / 100 \mathrm{ml}) \\
(n \text { 414) }\end{array}$ & $\begin{array}{l}\mathrm{IF}(n 416) \\
\mathrm{BF}(n 300)\end{array}$ & 1 year & No info & NS \\
\hline $\begin{array}{l}\text { Closa-Monasterolo } \\
\text { et al. }{ }^{(28)} \text { (Spain)† }\end{array}$ & Newborns & $\begin{array}{l}\text { Oligofructose-enriched inulin (SYN1) } \\
(0.8 \mathrm{~g} / 100 \mathrm{ml})(n 128)\end{array}$ & $\begin{array}{l}\mathrm{IF}(n 124) \\
\mathrm{BF}(n 136)\end{array}$ & 4 months & $\begin{array}{l}\text { BENEO Institute (an } \\
\text { Initiative of BENEO } \\
\text { GmbH). }\end{array}$ & $\begin{array}{l}\text { Significantly higher frequency of } \\
\text { depositions at any time } \\
\text { month } 1, P<0.001 ; \text { month } 2, P<0.01 \text {; } \\
\text { month } 3, P<0.01 \text {; month } 4, P<0.05\end{array}$ \\
\hline Ashley et al. ${ }^{(5)}$ (USA)† & Healthy term infants & PDX/GOS $(0.4 \mathrm{~g} / 100 \mathrm{I})$ & $\mathrm{IF}(n$ 142) & $120 d$ & Unclear & $\begin{array}{l}\text { Higher frequency of stools in the prebiotic } \\
\text { (PDX/GOS)-supplemented group at } \\
\text { some time intervals }\end{array}$ \\
\hline Scalabrin et al. ${ }^{(26)}(\mathrm{USA}) \dagger$ & $\begin{array}{l}\text { Healthy term infants } \\
21-30 \mathrm{~d}\end{array}$ & PDX/GOS $(0.4 \mathrm{~g} / 100 \mathrm{ml})(n 100)$ & $\begin{array}{l}\mathrm{IF}(n 101) \\
\mathrm{BF}(n 88)\end{array}$ & $60 d$ & $\begin{array}{l}\text { Mead Johnson } \\
\text { Nutrition }\end{array}$ & $\begin{array}{l}\text { No differences between the groups in } \\
\text { stool frequency during all study periods }\end{array}$ \\
\hline Gastrointestinal infections & & & & & & \\
\hline $\begin{array}{l}\text { Paineau et al }{ }^{(12)} \text { (France)† } \\
\text { Ripoll et al. }{ }^{(13)} \text { (Spain) } \dagger\end{array}$ & $\begin{array}{l}\text { Healthy, term infants }<7 d \\
\text { Healthy infants } \\
4 \mathrm{mo} \pm 2 \text { weeks }\end{array}$ & $\begin{array}{l}\text { FOS } 0.4 \mathrm{~g} / 100 \mathrm{ml}(n 31) \\
\text { FOS }(0.5 \mathrm{~g} / \mathrm{ml})(n 38)\end{array}$ & $\begin{array}{l}\text { IF }\left(\begin{array}{ll}n & 27) \\
\text { IF }(n \text { 37) }\end{array}\right.\end{array}$ & $\begin{array}{l}4 \text { months } \\
6 \text { months }\end{array}$ & $\begin{array}{l}\text { No info } \\
\text { Syrallberia S.A.U. } \\
\text { Saragossa, Spain }\end{array}$ & $\begin{array}{l}\text { NS (diarrhoea number of days) } \\
\text { NS (diarrhoea and gastroenteritis) }\end{array}$ \\
\hline Sierra et al. ${ }^{(14)}$ (Spain) $\dagger$ & $\begin{array}{l}\text { Healthy term infants } \\
<2 \text { months }\end{array}$ & $\begin{array}{l}\text { GOS }(0.44 \mathrm{~g} / 100 \mathrm{ml} \text { in IF and } 0.50 \mathrm{~g} / \mathrm{dl} \\
\text { in FF) }(n \text { 188) }\end{array}$ & IF (n 177) & 12 months & Hero Group & $\begin{array}{l}\text { NS (episodes of diarrhoea per infant; } \\
\text { number of infants with at least one } \\
\text { episode of diarrhoea per year) }\end{array}$ \\
\hline Bruzzese et al. $^{(18)}(\text { Italy })^{*}$ & Healthy infants $15-120 \mathrm{~d}$ & $\begin{array}{l}\text { GOS/FOS }(0.4 \mathrm{~g} / 100 \mathrm{ml}) \\
\quad(\mathrm{n} \mathrm{169)}\end{array}$ & IF $(n$ 173) & 12 months & $\begin{array}{l}\text { An unrestricted grant } \\
\text { from } \\
\text { Numico Research, } \\
\text { Friedrichsdorf, } \\
\text { Germany }\end{array}$ & $\begin{array}{l}\text { Lower rate of diarrhoeal episodes/child } \\
(0.12 \text { (SE 0.04) v. } 0.29 \text { (SE 0.05), } \\
P=0.015) \text {, lower number of children with } \\
\text { at least } 1 \text { episode of acute diarrhoea } \\
10 / 96(10.4 \%) \text { v. } 26 / 109 ; P=0.01\end{array}$ \\
\hline $\begin{array}{l}\text { Ivakhnenko et al. } \\
\quad(\text { Ukraine }) \dagger\end{array}$ & Newborns & GOS/FOS $(0.8 \mathrm{~g} / 100 \mathrm{ml})(\mathrm{n} 80)$ & $\begin{array}{l}\mathrm{IF}(\mathrm{n} 80) \\
\mathrm{BF}(\mathrm{n} 80)\end{array}$ & $\begin{array}{l}2 \text { month (follow- } \\
\text { up } 18 \text { months) }\end{array}$ & $\begin{array}{l}\text { Pediatrics } \\
\text { Department of Lviv } \\
\text { National Medical } \\
\text { University, } \\
\text { state registration } \\
\text { number } \\
0108 \cup 101130 .\end{array}$ & $\begin{array}{l}\text { Lower incidence of Gl infections (0.28 } \\
\text { (sE 0.05) v. 0.78 (SE 0.12) episodes/ } \\
\text { child/18 months, MD }-0.5(95 \% \mathrm{Cl} \\
-0.53,-0.47) \text {. }\end{array}$ \\
\hline $\begin{array}{l}\text { van Stuijvenberg et al. }{ }^{(34)} \\
\text { (The Netherlands, } \\
\text { Austria, Switzerland, } \\
\text { Italy, Germany† }\end{array}$ & Healthy infants $<8$ weeks & $\underset{(\mathrm{n} 414)}{\mathrm{GOS} / \mathrm{FOS}}+\mathrm{AOS}(0.8 \mathrm{~g} / 100 \mathrm{ml})$ & $\begin{array}{l}\mathrm{IF}(n 416) \\
\mathrm{BF}(n 300)\end{array}$ & 1 year & Danone Research & $\begin{array}{l}\text { NS (number of episodes of } \\
\text { gastroenteritis) }\end{array}$ \\
\hline $\begin{array}{l}\text { van Stuijvenberg et al. } \\
\text { (The Netherlands, } \\
\text { Austria, Switzerland, } \\
\text { Italy, Germany) } \\
\text { follow-up as above† }\end{array}$ & Healthy infants $<8$ weeks & $\begin{array}{l}\text { GOS/FOS + AOS }(0 \cdot 8 \mathrm{~g} / 100 \mathrm{ml}) \\
(\mathrm{n} \mathrm{414})\end{array}$ & $\begin{array}{l}\mathrm{IF}(n 416) \\
\mathrm{BF}(n 300)\end{array}$ & 1 year & Danone Research & $\begin{array}{l}\text { NS: Febrile episodes due to diarrhoea: } \\
0.51(25-75 \text { percentile } 0.03-1.07) \mathrm{v} \text {. } \\
0.53(25-75 \text { percentile } 0.03-1.51), \\
P=0.22 \\
\text { Shorter duration of diarrhoea: } 1.0(25-75 \\
\text { percentile } 0.0-4.0) \quad v .2 .0(25-75 \\
\text { percentile } 0.0-7.0), P=0.01\end{array}$ \\
\hline $\begin{array}{l}\text { Respiratory tract infections } \\
\text { Sierra et al. }{ }^{(14)} 2015 \\
\quad \text { (Spain) } \dagger\end{array}$ & $\begin{array}{l}\text { Healthy term infants } \\
<2 \text { months }\end{array}$ & $\begin{array}{l}\text { GOS }(0.44 \mathrm{~g} / 100 \mathrm{ml} \text { in IF and } 0.50 \mathrm{~g} / \mathrm{dl} \\
\text { in FF) }(n 188)\end{array}$ & IF $(n$ 177) & 12 months & Hero Group & $\begin{array}{l}\text { NS: Number of URTI episodes and the } \\
\text { number of infants with recurrent URTI }\end{array}$ \\
\hline Bruzzese et al. ${ }^{(18)}$ (Italy) $\dagger$ & Healthy infants $15-120 d$ & $\begin{array}{l}\text { GOS/FÓS } \\
(n \text { 169) }\end{array}$ & $\mathrm{IF}(n$ 173) & 12 months & $\begin{array}{l}\text { An unrestricted grant } \\
\text { from } \\
\text { Numico Research, } \\
\text { Friedrichsdorf, } \\
\text { Germany }\end{array}$ & $\begin{array}{l}\text { Number of children with one episode of } \\
\text { URTI - NS } \\
\text { Number of children with more than } \\
3 \text { episodes of URTI-NS }\end{array}$ \\
\hline
\end{tabular}




\section{N British Journal of Nutrition}

\begin{tabular}{|c|c|c|c|c|c|c|}
\hline References (country) & $\begin{array}{l}\text { Participants (age at } \\
\text { enrolment) }\end{array}$ & Intervention & Comparison & $\begin{array}{l}\text { Duration of } \\
\text { intervention } \\
\text { (follow-up) }\end{array}$ & Funding & $\begin{array}{l}\text { Effect (prebiotic-supplemented formula } \\
\text { group } v \text {. unsupplemented formula group) }\end{array}$ \\
\hline $\begin{array}{l}\text { Ivakhnenko et al. }{ }^{(20)} \\
\text { (Ukraine)† }\end{array}$ & Newborns & GOS/FOS $(0.8 \mathrm{~g} / 100 \mathrm{ml})(\mathrm{n} 80)$ & $\begin{array}{l}\text { IF (n 80) } \\
\text { BF (n 80) }\end{array}$ & $\begin{array}{l}2 \text { months (follow- } \\
\text { up } 18 \text { months) }\end{array}$ & $\begin{array}{l}\text { Pediatrics } \\
\text { Department of Lviv } \\
\text { National Medical } \\
\text { University, } \\
\text { state registration } \\
\text { number } \\
\text { 0108U101130. }\end{array}$ & $\begin{array}{l}\text { Incidence of URT infections: } 2.81 \\
\text { (SE } 0.51) \text { v. } 5.78 \text { (SE 0.97) episodes/ } \\
\text { child per } 18 \text { months; MD }-2.97 \\
(95 \% \mathrm{Cl}-3.26,-2 \cdot 68)\end{array}$ \\
\hline $\begin{array}{l}\text { van Stuijvenberg et al. }{ }^{(34)} \\
\text { (The Netherlands, } \\
\text { Austria, Switzerland, } \\
\text { Italy, Germany)t }\end{array}$ & Healthy infants $<8$ weeks & $\begin{array}{l}\text { GOS/FOS + AOS }(0.8 \mathrm{~g} / 100 \mathrm{ml}) \\
(n \text { 414) }\end{array}$ & $\begin{array}{l}\text { IF (n 416) } \\
\text { BF (n 300) }\end{array}$ & 1 year & Danone Research & $\begin{array}{l}\text { URTI, ear infection, pneumonia/ } \\
\text { bronchiolitis - NS }\end{array}$ \\
\hline $\begin{array}{l}\text { van Stuijvenberg et al. }{ }^{(31)} \\
\text { (The Netherlands, } \\
\text { Austria, Switzerland, } \\
\text { Italy, Germany) } \\
\text { follow-up as above† } \\
\text { Allergic diseases }\end{array}$ & Healthy infants $<8$ weeks & $\begin{array}{l}\text { GOS/FOS + AOS }(0 \cdot 8 \mathrm{~g} / 100 \mathrm{ml}) \\
(\mathrm{n} \mathrm{414)}\end{array}$ & $\begin{array}{l}\mathrm{IF}(n 416) \\
\mathrm{BF}(n 300)\end{array}$ & 1 year & Danone Research & $\begin{array}{l}\text { Episodes and duration of coughing, } \\
\text { wheezing, and blocked nose at } 3 \text { to } \\
5 \text { years - NS }\end{array}$ \\
\hline Sierra et al. ${ }^{(14)}$ (Spain)† & $\begin{array}{l}\text { Healthy term infants } \\
<2 \text { months }\end{array}$ & $\begin{array}{l}\text { GOS }(0.44 \mathrm{~g} / 100 \mathrm{ml} \text { in } \mathrm{IF} \text { and } 0.50 \mathrm{~g} / \mathrm{dl} \\
\text { in FF) }(n 188)\end{array}$ & $\mathrm{IF}(n 177)$ & 12 months & Hero Group & $\begin{array}{l}\text { Allergic manifestations and allergic } \\
\text { sensitisation - NS }\end{array}$ \\
\hline $\begin{array}{l}\text { Ivakhnenko et al. } \\
\text { (Ukraine) } \dagger\end{array}$ & Newborns & GOS/FOS $(0.8 \mathrm{~g} / 100 \mathrm{ml})(n 80)$ & $\begin{array}{l}\mathrm{IF}(n 80) \\
\mathrm{BF}(n 80)\end{array}$ & $\begin{array}{l}2 \text { months (follow- } \\
\text { up } 18 \text { months) }\end{array}$ & $\begin{array}{l}\text { Pediatrics } \\
\text { Department of Lviv } \\
\text { National Medical } \\
\text { University, } \\
\text { state registration } \\
\text { number } \\
\text { 0108U101130. }\end{array}$ & $\begin{array}{l}\text { Allergic reactions to food products: } \\
3 / 62 \text { v. } 9 / 53 \text {, RR } 0.28(95 \% \mathrm{Cl} 0.09, \\
0.9) \\
\text { Allergic reactions to cows' milk protein: } \\
2 / 62 \text { v. } 8 / 53 \text {, RR } 0.2(95 \% \mathrm{Cl} 0.05, \\
0.84) \\
\text { Atopic dermatitis: } 3 / 62 \text { v. } 9 / 53 \text {, RR } 0.28 \\
\text { (95\% Cl } 0.09,0.9) \\
\text { Respiratory allergic symptoms (recurrent } \\
\text { wheezing): } 3 / 62 \text { v. } 7 / 53 \text {, RR } 0.36 \\
\text { (95\% Cl } 0.1,1.2) \\
\text { Gl food allergy: } 2 / 62 \text { v. } 7 / 63, \text { RR } 0.24 \\
(95 \% \mathrm{Cl} 0.06,0.98) \text {. }\end{array}$ \\
\hline $\begin{array}{l}\text { Grüber et al. } .^{(35)} \text { (the } \\
\text { Netherlands, Austria, } \\
\text { Switzerland, Italy } \\
\text { Germany } \\
\text { (The same population as } \\
\text { van Stuijvenberg) } \dagger\end{array}$ & healthy infants $<8$ weeks & $\begin{array}{l}\text { GOS/ FOS + AOS }(0.8 \mathrm{~g} / 100 \mathrm{ml}) \\
(n \text { 414) }\end{array}$ & $\begin{array}{l}\mathrm{IF}(n 416) \\
\mathrm{BF}(n 300)\end{array}$ & 12 months & $\begin{array}{l}\text { Danone Research, } \\
\text { Friedrichsdorf, } \\
\text { Germany }\end{array}$ & Atopic dermatitis: 5.7 v. $9.7 \% ; P=0.04$. \\
\hline \multicolumn{7}{|l|}{ Antibiotic therapy } \\
\hline Ripoll et al. ${ }^{(13)}$ (Spain)† & $\begin{array}{l}\text { Healthy infants } \\
4 \text { months } \pm 2 \text { weeks }\end{array}$ & FOS $(0.5 \mathrm{~g} / \mathrm{ml})(n 38)$ & IF ( $n$ 37) & 6 months & $\begin{array}{l}\text { Syrallberia S.A.U. } \\
\text { Saragossa, Spain }\end{array}$ & $\begin{array}{l}\text { Number of infants with concomitant } \\
\text { treatments (including antibiotics) - NS } \\
(P=0.12)\end{array}$ \\
\hline Sierra et al. ${ }^{(14)}$ (Spain)† & $\begin{array}{l}\text { Healthy term infants } \\
<2 \text { months }\end{array}$ & $\begin{array}{l}\text { GOS }(0.44 \mathrm{~g} / 100 \mathrm{ml} \text { in IF and } 0.50 \mathrm{~g} / \mathrm{dl} \\
\text { in FF) }(n 188)\end{array}$ & IF $(n$ 177) & 12 months & Hero Group & NS \\
\hline Bruzzese et al. ${ }^{(18)}$ (Italy) $)^{\star}$ & Healthy infants $15-120 \mathrm{~d}$ & $\underset{(n \text { 169) }}{\operatorname{GOS} / \mathrm{FOOS}}(0.4 \mathrm{~g} / 100 \mathrm{ml})$ & IF $(n$ 173) & 12 months & $\begin{array}{l}\text { An unrestricted grant } \\
\text { from } \\
\text { Numico Research, } \\
\text { Friedrichsdorf, } \\
\text { Germany }\end{array}$ & $\begin{array}{l}\text { Significantly lower mean rate of antibiotic } \\
\text { Courses: } 1.03(\mathrm{SE} 0.15) \text { v. } 1.48 \text { (SE } \\
0.16 \text { ); MD } 0.45,95 \% \mathrm{Cl}-0.49,-0.4 ; \\
P=0.038 ; \\
\text { Multiple antibiotic courses/year: } 24 / 60 \\
\text { V. } 43 / 65 ; P=0.004\end{array}$ \\
\hline $\begin{array}{l}\text { van Stuijvenberg et al. }{ }^{(34)} \\
\text { (The Netherlands, } \\
\text { Austria, Switzerland, } \\
\text { Italy, Germany)† }\end{array}$ & Healthy infants $<8$ weeks & $\begin{array}{l}\text { GOS/FOS + AOS }(0 \cdot 8 \mathrm{~g} / 100 \mathrm{ml}) \\
(n \text { 414) }\end{array}$ & $\begin{array}{l}\text { IF }(n+416) \\
\operatorname{BF}(n \text { 300) }\end{array}$ & 1 year & Danone Research & $\begin{array}{l}\text { NS: number of fever episodes requiring } \\
\text { systemic antibiotics during the } 1 \text { st year } \\
\text { of life }\end{array}$ \\
\hline
\end{tabular}




\begin{tabular}{|c|c|c|c|c|c|c|}
\hline References (country) & $\begin{array}{l}\text { Participants (age at } \\
\text { enrolment) }\end{array}$ & Intervention & Comparison & $\begin{array}{l}\text { Duration of } \\
\text { intervention } \\
\text { (follow-up) }\end{array}$ & Funding & $\begin{array}{l}\text { Effect (prebiotic-supplemented formula } \\
\text { group } v \text {. unsupplemented formula group) }\end{array}$ \\
\hline $\begin{array}{l}\text { van Stuijvenberg et al. }{ }^{(31)} \\
\text { (The Netherlands, } \\
\text { Austria, Switzerland, } \\
\text { Italy, Germany) } \\
\text { follow-up as above† } \\
\text { Adverse events }\end{array}$ & Healthy infants $<8$ weeks & $\begin{array}{l}\text { GOS/FOS + AOS }(0.8 \mathrm{~g} / 100 \mathrm{ml}) \\
(n \text { 414) }\end{array}$ & $\begin{array}{l}\mathrm{IF}(n 416) \\
\mathrm{BF}(n 300)\end{array}$ & 1 year & Danone Research & $\begin{array}{l}\text { NS: number of fever episodes requiring } \\
\text { systemic antibiotics at } 3 \text { and } 5 \text { years } \\
\text { follow-up }\end{array}$ \\
\hline Bettler-Euler et al..$^{(8)}($ USA)* & Healthy term infants & $\begin{array}{l}\text { FOS }(0.3 \mathrm{~g} / 100 \mathrm{ml})(\mathrm{n} 101) \\
\text { FOS }(0.15 \mathrm{~g} / 100 \mathrm{ml})(\mathrm{n} \mathrm{98})\end{array}$ & IF $(n$ 98) & 12 weeks & No info & $\begin{array}{l}0.3 \mathrm{~g} / 100 \mathrm{ml} v .0 .15 \mathrm{~g} / 100 \mathrm{ml} \text { : reduced } \\
\text { constipation }(P=0.03) \text {, increased } \\
\text { vomiting }(P=0.016) \text {. } \\
\text { Flatulence, diarrhoea, loose stools, } \\
\text { dehydration or allergic reaction - NS }\end{array}$ \\
\hline Brunser et al. $^{(9)}(\text { Chile })^{\star}$ & $\begin{array}{l}\text { Healthy term infants, } \\
\text { enrolled at } 3.5 \text { months }\end{array}$ & $\begin{array}{l}\text { FOS }(0.2 \mathrm{~g} / 100 \mathrm{ml})\left(\begin{array}{ll}n & 32\end{array}\right) \\
\text { L johnsonii La1 } 10^{8} \mathrm{CFU} / \mathrm{g}(n 25)\end{array}$ & $\begin{array}{l}\mathrm{FF}\left(\begin{array}{ll}n 3 \\
\mathrm{BF}\end{array}(n 26)\right. \\
\mathrm{B} 26\end{array}$ & $\begin{array}{l}13 \text { weeks } \\
(15 \text { weeks })\end{array}$ & No info & $\begin{array}{l}\text { The number of adverse events per infant, } \\
\text { including upper and lower respiratory } \\
\text { infections and diarrhoeal episodes - } \\
\text { NS }(P>0.05 \text {; data not shown) }\end{array}$ \\
\hline Paineau et al. ${ }^{(12)}$ (France) $\dagger$ & Healthy, term infants $<7 d$ & FOS $0.4 \mathrm{~g} / 100 \mathrm{ml}(n 31)$ & $\mathrm{IF}(n 27)$ & 4 months & No info & $\begin{array}{l}\text { NS (not related to the tested formula } \\
\text { (bronchiolitis)) }\end{array}$ \\
\hline Ripoll et al. ${ }^{(13)}$ (Spain)† & $\begin{array}{l}\text { Healthy infants } \\
4 \text { months } \pm 2 \text { weeks }\end{array}$ & FOS $(0.5 \mathrm{~g} / \mathrm{ml})(n 38)$ & IF ( $n$ 37) & 6 months & $\begin{array}{l}\text { Syrallberia S.A.U. } \\
\text { Saragossa, Spain }\end{array}$ & NS (total AEs, diarrhoea, gastroenteritis) \\
\hline $\begin{array}{l}\text { Wernimont et al. }{ }^{\left({ }^{2}\right)} \\
\text { (USA) } \dagger\end{array}$ & $\begin{array}{l}\text { Healthy term infants } \\
5-15 d\end{array}$ & FOS $(0.3 \mathrm{~g} / 100 \mathrm{ml})(n 47)$ & $\begin{array}{l}\mathrm{IF}(n 48) \\
\mathrm{BF}(n 50)\end{array}$ & 8 weeks & Wyeth Nutrition & $\begin{array}{l}\text { Total AE: } 15 / 19 \text { v. } 13 / 20 \text { (RR 1.2; } 95 \% \mathrm{Cl} \\
\text { 0.8, 1.8, NS); } \\
\text { Feeding-related GI AEs: } 13 / 19 \mathrm{v} .13 / 20 \\
\text { (RR 1.0; } 95 \% \mathrm{Cl} 0.7,1.6, \mathrm{NS} \text { ) } \\
\text { Withdrawals (vomiting, spitting up, and } \\
\text { abdominal pain) } 7 / 19 \mathrm{v} .2 / 20 \text { (RR } 3.7 \text {; } \\
\quad 95 \% \mathrm{Cl} 0.9,15 \cdot 6, \mathrm{NS} \text { ) }\end{array}$ \\
\hline Ashley et al. ${ }^{(5)}$ (USA)† & Healthy term infants & GOS $(0.4 \mathrm{~g} / 100 \mathrm{ml})$ & IF $(n$ 142) & $120 d$ & Unclear & $\begin{array}{l}\text { Incidence of at least one medically } \\
\text { confirmed } A E, 77 v .78 \%-N S \text {. } \\
\text { Eyes, ears, nose, and throat system - } \\
\text { NS } \\
\text { GI reflux, emesis, or diarrhoea - NS } \\
\text { Excessive spitting - significantly higher in } \\
\text { GOS group }(P<0.05) \\
\text { SAEs (all unrelated to study formulas) - } \\
\text { NS }\end{array}$ \\
\hline Ben et al. ${ }^{(6,7)}(\mathrm{China})^{*}$ & Term infants & GOS $(0.24 \mathrm{~g} / 100 \mathrm{ml})(n 69)$ & $\begin{array}{l}\mathrm{IF}(n 52) \\
\mathrm{BF}(n 26)\end{array}$ & 6 months & No info & NS (crying, regurgitation, vomiting) \\
\hline Bisceglia et al. ${ }^{(16)}$ (Italy) $\dagger$ & Healthy term infants & GOS/FOS $(0.8 \mathrm{~g} / 100 \mathrm{ml})(n 39)$ & IF $(n 37)$ & $28 \mathrm{~d}$ & $\begin{array}{l}\text { Partially financed by } \\
\text { Numico } \\
\text { Friedrichsdorf } \\
\text { Germany }\end{array}$ & NS (none) \\
\hline Costalos et al. ${ }^{(17)}$ (Greece) & $\begin{array}{l}\text { Healthy term infants, } \\
\text { enrolled } \leq 14 \mathrm{~d}\end{array}$ & GOS/FOS $(0.4 \mathrm{~g} / 100 \mathrm{ml})(n 80)$ & IF $(n 80)$ & 12 weeks & No info & NS \\
\hline Giovannini et al. ${ }^{(11)}$ (Italy) $\dagger$ & $<15 d$ & GOS $(0.4 \mathrm{~g} / 100 \mathrm{ml})(n 83)$ & $\begin{array}{l}\mathrm{IF}(n 80) \\
\mathrm{BF}(n 199)\end{array}$ & 6 months & No info & $\begin{array}{l}\text { Diarrhoeal, crying, and vomiting episodes } \\
-\mathrm{NS} \\
\text { Infantile colic - NS or reduced } \\
\text { (inconsistent results) } \\
\text { Higher stool frequency } P<0.05 \\
\text { Regurgitation } P<0.05\end{array}$ \\
\hline Matsuki et al. ${ }^{(33)}$ (Japan)† & $\begin{array}{l}\text { Healthy term infants } \\
31-54 \mathrm{~d}\end{array}$ & GOS $(0.3 \mathrm{~g} / 100 \mathrm{ml})(n 17)$ & $\mathrm{IF}(n 18)$ & 2 weeks & $\begin{array}{l}\text { Yakult Central } \\
\text { Institute }\end{array}$ & $\begin{array}{l}\text { Regurgitations, gassiness, or rejections - } \\
\text { no reported }\end{array}$ \\
\hline $\begin{array}{l}\text { Piemontese et al. }{ }^{(24)} \text { (Italy, } \\
\text { Switzerland, Germany, } \\
\text { Austria, the Netherlands) } \\
\text { (The same population as } \\
\text { van Stuijvenberg) } \dagger\end{array}$ & $\begin{array}{l}\text { Healthy, term infants } \\
<8 \text { weeks }\end{array}$ & $\begin{array}{l}\text { GOS/FOS + AOS }(0.8 \mathrm{~g} / 100 \mathrm{ml}) \\
(n \text { 414) }\end{array}$ & $\begin{array}{l}\mathrm{IF}(n 416) \\
\mathrm{BF}(n 300)\end{array}$ & 1 year & No info & $\begin{array}{l}\text { AE (otitis media, bronchitis, } \\
\text { gastroenteritis, URTI, varicella, } \\
\text { bronchiolitis, pharyngitis, UTI): } 31 \mathrm{v} \text {. } \\
30 \% ; P>0.05 \text { ) } \\
\text { SAEs: } 10.6 \text { v. } 9.4 \% \text {; NS }\end{array}$ \\
\hline
\end{tabular}




\section{Nes British Journal of Nutrition}

\begin{tabular}{|c|c|c|c|c|c|c|}
\hline References (country) & $\begin{array}{l}\text { Participants (age at } \\
\text { enrolment) }\end{array}$ & Intervention & Comparison & $\begin{array}{l}\text { Duration of } \\
\text { intervention } \\
\text { (follow-up) }\end{array}$ & Funding & $\begin{array}{l}\text { Effect (prebiotic-supplemented formula } \\
\text { group } v \text {. unsupplemented formula group) }\end{array}$ \\
\hline Salvini et al. ${ }^{(22)}$ (Italy) $\dagger$ & $\begin{array}{l}\text { Healthy newborns of } \\
\text { hepatitis C virus-infected } \\
\text { mothers }\end{array}$ & GOS/FOS $(0.8 \mathrm{~g} / 100 \mathrm{ml})(n 10)$ & $\mathrm{IF}(n \mathrm{10})$ & 6 months & $\begin{array}{l}\text { Danone Research, } \\
\text { Centre for } \\
\text { Specialised } \\
\text { Nutrition }\end{array}$ & No AE recorded \\
\hline $\begin{array}{l}\text { Scholtens et al. }{ }^{(36)} \\
\text { (Belgium) }\end{array}$ & Healthy term infants & GOS/FOS $(0.6 \mathrm{~g} / 100 \mathrm{ml})(n 27)$ & $\begin{array}{l}\mathrm{IF}(n 29) \\
\operatorname{BF}(n 31 / 39)\end{array}$ & $\begin{array}{l}6 \text { months } \\
(26 \text { weeks })\end{array}$ & $\begin{array}{l}\text { Numico Research, } \\
\text { Wageningen, The }\end{array}$ & NS \\
\hline $\begin{array}{l}\text { van Stuijvenberg et al. } \\
\text { (The Netherlands, } \\
\text { Austria, Switzerland, } \\
\text { Italy, Germany)* }\end{array}$ & Healthy infants $<8$ weeks & $\begin{array}{l}\text { GOS/FOS }+ \text { AOS }(0.8 \mathrm{~g} / 100 \mathrm{ml}) \\
(n \text { 414) }\end{array}$ & $\begin{array}{l}\mathrm{IF}(n 416) \\
\mathrm{BF}(n 300)\end{array}$ & 1 year & Danone Research & No serious $\mathrm{AE}$ related to the intervention \\
\hline $\begin{array}{l}\text { Veereman-Wauters } \\
\text { et al. }{ }^{(23)} \text { (Belgium)† }\end{array}$ & Healthy term infants $<5 d$ & $\begin{array}{l}\text { GOS/FOS }(0.8 \mathrm{~g} / 100 \mathrm{ml}) \\
\text { Oligofructose-enriched inulin SYN1 } \\
\text { (0.4 or } 0.8 \mathrm{~g} / 100 \mathrm{ml})\end{array}$ & $\begin{array}{l}\mathrm{IF}(n 21) \\
\mathrm{BF}(n 29)\end{array}$ & $28 d$ & Beneo-Orafti & No SAE related to the intervention \\
\hline Williams et al. $^{(15)}$ (USA)† & Healthy term $<8 d$ & $\begin{array}{l}\text { GOS }(0.4 \mathrm{~g} / 100 \mathrm{ml})\left(\begin{array}{ll}n \\
\text { GOS }\end{array}(0.8 \mathrm{~g} / 100 \mathrm{ml})(n \text { } n 3)\right.\end{array}$ & IF ( $n$ 44) & $119 d$ & Abbott Nutrition & $\begin{array}{l}\text { Higher proportion of SAE, primarily } \\
\text { respiratory tract infections }(P<0.05)\end{array}$ \\
\hline $\begin{array}{l}\text { Closa-Monasterolo } \\
\text { et al. }{ }^{(28)} \text { (Spain) } \dagger\end{array}$ & Newborns & $\begin{array}{l}\text { Oligofructose-enriched inulin (SYN1) } \\
(0.8 \mathrm{~g} / 100 \mathrm{ml})(n \text { 128) }\end{array}$ & $\begin{array}{l}\mathrm{IF}(n 124) \\
\mathrm{BF}(n \text { 136) }\end{array}$ & 4 months & $\begin{array}{l}\text { BENEO Institute (an } \\
\text { Initiative of BENEO } \\
\text { GmbH). }\end{array}$ & $\begin{array}{l}\text { Regurgitation, digestive discomfort }-N S \\
\text { Crying (min/d) - NS (except at } 4 \text { months - } \\
\text { longer in the prebiotic group; } P<0.05 \text { ) } \\
\text { Total AE: NS (except less loose stools in } \\
\text { the prebiotic group: } 2 \text { v. } 8 \%, P<0.05 \text { ) }\end{array}$ \\
\hline Ashley et al. ${ }^{(5)}($ USA)† & Healthy term infants & PDX/GOS $(0.4 \mathrm{~g} / 100 \mathrm{ml})$ & IF $(n$ 142) & $120 \mathrm{~d}$ & Unclear & \\
\hline Nakamura et al. ${ }^{(25) *}$ & $\begin{array}{l}\text { Healthy term infants } 13 \text { to } \\
92 \mathrm{~d}\end{array}$ & PDX/GOS $(0.4 \mathrm{~g} / 100 \mathrm{ml})$ & $\begin{array}{l}\mathrm{IF}(n 25) \\
\mathrm{BF}(n 30)\end{array}$ & $28 d$ & $\begin{array}{l}\text { Mead Johnson \& } \\
\text { Company, } \\
\text { Evansville, IN }\end{array}$ & NS (data not shown) \\
\hline Scalabrin et al. ${ }^{(26)}(\mathrm{USA}) \dagger$ & $\begin{array}{l}\text { Healthy term infants } \\
21-30 \mathrm{~d}\end{array}$ & PDX/GOS $(0.4 \mathrm{~g} / 100 \mathrm{ml})(n 100)$ & $\begin{array}{l}\mathrm{IF}(n 101) \\
\mathrm{BF}(n 88)\end{array}$ & $60 d$ & $\begin{array}{l}\text { Mead Johnson } \\
\text { Nutrition }\end{array}$ & $\begin{array}{l}\text { NS: } 1 \text { or more AE or when analysed by } \\
\text { type of event }\end{array}$ \\
\hline Ziegler et al. ${ }^{(27)}(\mathrm{USA})^{*}$ & Healthy term infants $<14 \mathrm{~d}$ & $\begin{array}{l}\mathrm{PDX}+\mathrm{GOS}(0.4 \mathrm{~g} / 100 \mathrm{ml})(n 74) \\
\text { PDX + GOS + LOS }(0.8 \mathrm{~g} / 100 \mathrm{ml}) \\
(n 76)\end{array}$ & $\mathrm{IF}(n 76)$ & $120 \mathrm{~d}$ & Mead Johnson \& Co. & $\begin{array}{l}\text { Serious AE (none related to the study } \\
\text { products): NS } \\
\text { Diarrhoea (control v. PG4, } 4 \text { v. } 18 \% \text {, } \\
P=0.008 \text { ), } \\
\text { Eczema (PG4 v. control, } 18 \text { v. } 7 \% \text {, } \\
\text { P.0.046; PG4 v. PGL8, } 18 \text { v. } 4 \% \text {, } \\
P=0.008 \text { ), } \\
\text { Irritability (control v. PGL8, } 4 \text { v. } 16 \% \text {, } \\
P=0.027 \text { ) }\end{array}$ \\
\hline Nakamura et al. ${ }^{(25) \star}$ & $\begin{array}{l}\text { Healthy term infants } 13 \text { to } \\
92 \mathrm{~d}\end{array}$ & PDX/GOS $(0.4 \mathrm{~g} / 100 \mathrm{ml})$ & $\begin{array}{l}\mathrm{IF}(n 25) \\
\mathrm{BF}(n 30)\end{array}$ & $28 d$ & $\begin{array}{l}\text { Mead Johnson \& } \\
\text { Company, } \\
\text { Evansville, IN }\end{array}$ & NS (data not shown) \\
\hline
\end{tabular}

GOS, short-chain galactooligosaccharides; FOS, long-chain fructo-oligosaccharides; AOS, acidic oligosaccharides; IF, infant formula; BF, breast-feeding; BAE, adverse event; FF, follow-on formula; SYN1, 50:50 mixture of long chain inulin (Orafti HP) and oligofructose (b(2-1)-linked fructo-oligosaccharides with less than nine fructose moieties and partially containing a terminal glucose unit; PDX, polydextrose; LOS, lactulose; CFU, colony-forming units; Gl, gastrointestinal; SAE, serious adverse event; URTI, upper respiratory tract infection; UTI, urinary tract infection.

* The effects as reported in a 2011 review (for details, see 1).

† Newly identified evidence (for details, see text). 


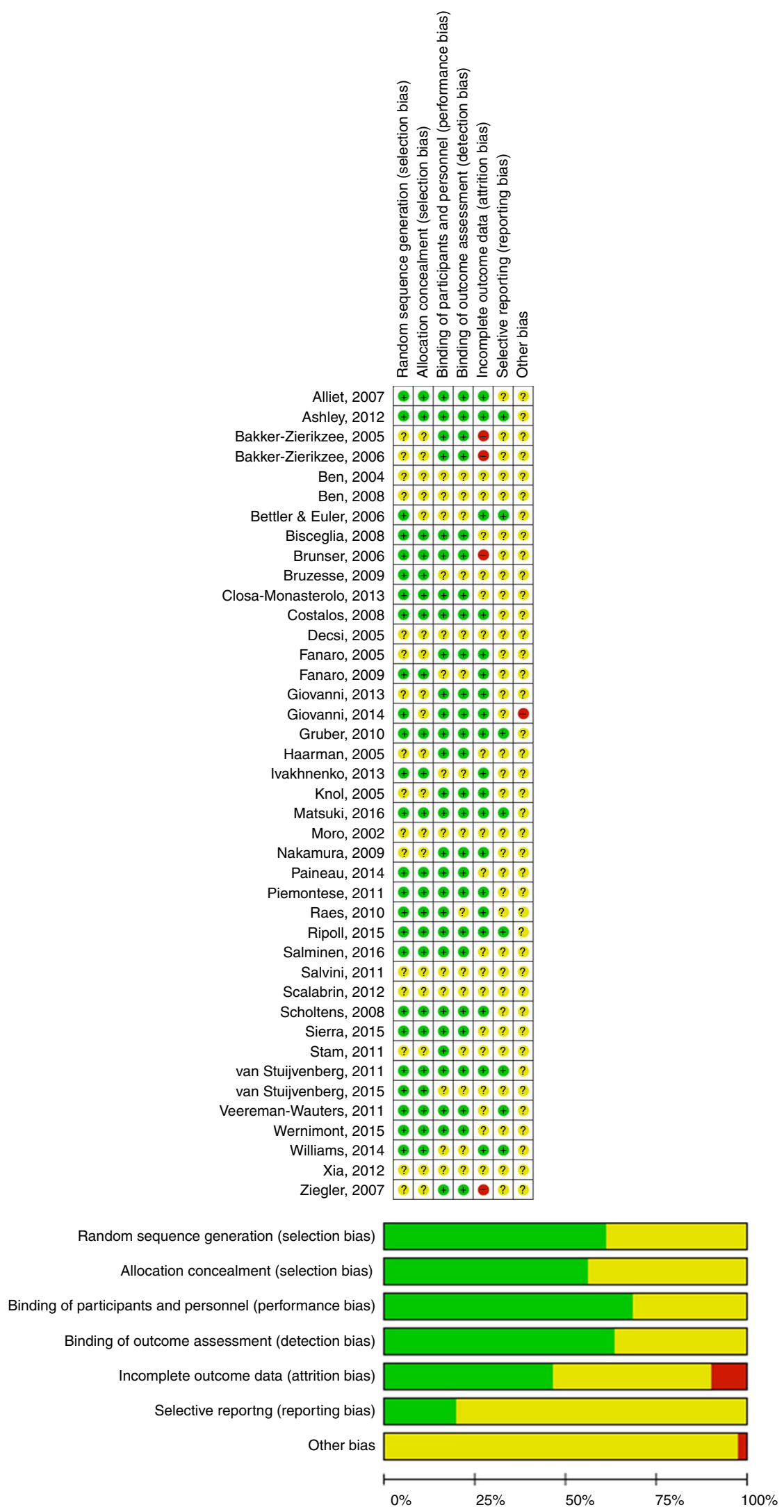

Fig. 1. Assessment of the risk of bias in included trials and the review authors' judgements about each risk of bias item presented as percentages across all included studies. $\square$, Low risk of bias; $\square$, unclear risk of bias; $\square$, high risk of bias. For a colour figure, see the online version of the paper. 


\section{Clinical effects}

Below, we summarise evidence from trials reporting clinical outcomes that have not been included in the earlier report by the ESPGHAN Committee on Nutrition ${ }^{(1)}$.

Growth. A number of trials assessed the effects of prebiotic supplementation on growth, including FOS supplementation of infant formula, either $0.4 \mathrm{~g} / 100 \mathrm{ml}^{(12)}$ or $0.5 \mathrm{~g} / 100 \mathrm{ml},{ }^{(13)}$ and GOS supplementation (with various GOS contents) ${ }^{(5,10,11,14,15)}$. In both cases, there were no significant differences between the prebioticsupplemented and the unsupplemented formula groups. In 4 studies investigating the effects of the administration of formula supplemented with $0.8 \mathrm{~g} / 100 \mathrm{ml}$ of GOS/FOS, no significant differences between the prebiotic and control groups in anthropometric parameters (weight, length and head circumference) were found at any time $e^{(16,20,22,23)}$. However, in a study by Bruzzese et al. the authors observed a transient increase in body weight. In the GOS/FOS-supplemented formula group compared with the control formula group, there was a significant increase in the mean body weight at 3 and 6 months of follow-up $(P<0 \cdot 01)$, whereas it was similar in the two groups at 9 and 12 months of follow-up. Mean body length was significantly greater in the GOS/ FOS-supplemented formula group at all-time intervals $(P<0.05)$, whereas mean head circumference was similar in the two groups at 3, 6, 9 and 12 months of follow-up (data not shown) ${ }^{(18)}$. The supplementation with FOS/GOS $+\operatorname{AOS}^{(24)}$ and the supplementation with two different doses of oligofructose-enriched inulin $(0.4 \mathrm{~g} / 100 \mathrm{ml} \text { and } 0.8 \mathrm{~g} / 100 \mathrm{ml} \text { supplementation })^{(23,28)}$ had no significant effects on growth. Similarly, the supplementation with $\mathrm{PDX} / \mathrm{GOS}^{(5,26)}$ resulted in no differences in anthropometric measures between infants receiving a control formula or a formula supplemented with $0.4 \mathrm{~g} / 100 \mathrm{ml}$ of a prebiotic blend of PDX and GOS from 14 to $60 \mathrm{~d}$ of age ${ }^{(26)}$ or from 14 to $120 \mathrm{~d}$ of age ${ }^{(5)}$.

Tolerance. Several new studies evaluated the influence of prebiotic-supplemented formulae on tolerance. Although there were different definitions of tolerance, there were no significant differences in tolerance between infants who received unsupplemented and prebiotic-supplemented formulae in the majority of studies. There were no differences in the number of days of abdominal pain (with crying) (0.22 (SE 0.28) v. $0 \cdot 23$ (SE 0.23), respectively; $P$ value not shown) or nausea $(0.05$ (SE 0.12$) v \cdot 0.03$ (se 0.07), respectively, $P$ value not shown) between infants who received unsupplemented or FOS-supplemented $(0.4 \mathrm{~g} / 100 \mathrm{ml})$ formula $^{(12)}$, no significant differences between infants fed FOS-supplemented formulae $(0.2 \mathrm{~g} / 100 \mathrm{ml}$ or $0.3 \mathrm{~g} / 100 \mathrm{ml})$ or unsupplemented formula in the frequency of spitting up or vomiting (no exact data shown) ${ }^{(29)}$, and no significant differences between infants fed FOS-supplemented formula $(0.5 \mathrm{~g} / 100 \mathrm{ml})$ or unsupplemented formula in the mean number of days with regurgitation (30.0 (SE 5.8) v. 27.7 (SE 5.0), respectively; $P=0.79$ ), crying (20.7 (SE 6.0) v. 18.67 (SE 5.2), respectively; $P=0.85$ ), and constipation (18.2 (SE 4.7) v. 9.8 (SE 2.4 ), respectively; $P=0.23$ ). However, there was a significantly reduced number of days with vomiting $(4 \cdot 1(\operatorname{se} 0.7) v .7 .7(\operatorname{se~} 1.3) ; P=0.05)$ in the FOSsupplemented formula group ${ }^{(13)}$. No significant differences were found in tolerance symptoms, such as fussiness and gassiness, between an unsupplemented formula group and either a GOSsupplemented formula $(0.4 \mathrm{~g} / 100 \mathrm{ml})$ group $^{(5)}$ or a PDX/GOSsupplemented formula group ${ }^{(5,26)}$. Regurgitation and vomiting scores were evaluated in infants receiving GOS/FOS- or oligofructose-enriched inulin-supplemented formulae ${ }^{(23)}$, with no significant differences found between the supplemented and unsupplemented formula groups. Tolerance was also defined as the incidence of episodes of crying, regurgitation, vomiting and flatulence. No differences between groups were found in a studies comparing GOS- $^{(10)}$ (no data shown), GOS/FOS- and AOS- $(0 \cdot 8 \mathrm{~g} / 100 \mathrm{ml})$ supplemented formulae with unsupplemented formula $(P>0.05)^{(24)}$. There were also no differences in either the duration of vomiting (median 2.0 (25-75 centile $0 \cdot 0-4 \cdot 0) v \cdot 2 \cdot 0$ (25-75 centile 0.0-5.0), respectively; $P=0 \cdot 13)$ or the number of episodes of vomiting (median 0.87 (25-75 centile 0.4-1.56) v. 0.91 (25-75 centile 0.9-1.55), respectively; $P=0.46)$ in the supplemented formula group compared with the unsupplemented formula group ${ }^{(31)}$.

Stool frequency. Stool frequency was evaluated in twelve new trials. Although different prebiotics were used in the majority of studies, there were no significant effects on stool frequency between prebiotic-supplemented and unsupplemented formula groups, including FOS supplementation (two RCT) ${ }^{(29,32)}$, GOS supplementation (five RCT) ${ }^{(10,15,33)}$, FOS/GOS supplementation ${ }^{(23)}$, FOS/GOS/AOS supplementation ${ }^{(24)}$, oligofructose-enriched inulin supplementation $^{(23)}$ and PDX/GOS supplementation ${ }^{(26)}$. Only four studies reported a higher frequency of stools in the prebioticsupplemented formula group, either at all study time points (FOS/GOS) $^{(16)}$ and (oligofructose-enriched inulin) ${ }^{(28)}$ or at some time intervals (GOS at 7, 14 and $60 \mathrm{~d}$ ), ${ }^{(5)}$ (GOS at 3 and 4 months) ${ }^{(14)}$ and (PDX/GOS at 7, 14 and $\left.60 \mathrm{~d}\right)^{(5)}$.

Stool consistency. A number of new trials assessed the effects of prebiotic supplementation on stool consistency, including FOS supplementation (three RCT) ${ }^{(13,29,32)}$, GOS supplementation (four RCT) ${ }^{(5,10,14,15)}$, FOS/GOS supplementation (two RCT) ${ }^{(18,23)}$, FOS/GOS/AOS supplementation (one RCT) ${ }^{(24)}$, PDX/GOS supplementation (two RCT) ${ }^{(5,26)}$ and oligofructoseenriched inulin supplementation (two RCT $)^{(23,28)}$. These trials documented a softening effect of prebiotic supplementation of formula on stool consistency (Table 1).

Gastrointestinal infections/diarrhoea. Six new studies evaluated the effects of prebiotic supplementation on the incidence of episodes of diarrhoea/gastrointestinal infections. Neither addition of FOS, GOS, nor FOS/GOS/AOS had an influence on the frequency of these episodes, with no differences between the supplemented and unsupplemented formula groups. Paineau et $a l^{(12)}$ found no difference between control and FOSsupplemented groups in the number of days with diarrhoea, defined as the number of days with liquid stools $(0 \cdot 10$ (SE 0.16) $v$. $0 \cdot 18$ (SE 0.24 ), respectively; $P$ value not shown). There were no significant differences between unsupplemented and GOS-supplemented formula groups in either the number of episodes of diarrhoea (defined as semiliquid or liquid faeces in 
three or more depositions per day for at least $3 \mathrm{~d}$ ) per infant (0.20 (SE 0.52) v. 0.27 (SE 0.67); respectively; MD 0.07, 95\% CI $-0.05,0.19 ; P=0.36)$ or the number of infants with at least 1 episode of diarrhoea/year (15.9 $v$. 18.2\%, respectively; $P=0 \cdot 8)^{(14)}$. There were also no significant differences in the number of episodes of gastroenteritis (diagnosed as a fever episode accompanied by vomiting and diarrhoea, or only diarrhoea) between infants receiving either standard or follow-up formula supplemented with $0 \cdot 8 \mathrm{~g} / 10 \mathrm{ml}$ of GOS/FOS/ AOS or control formula during the first 12 months of the intervention $^{(34)}$, as well as in the adjusted frequency of febrile episodes due to diarrhoea analysed prospectively at 3-5 years (0.51 (25-75 centile 0.03-1.07) v. 0.53 (25-75 centile 0.03-1.51), respectively; $P=0.22)$; however, the authors reported a shorter duration $(d)$ of diarrhoea in the experimental group ( 1.0 (25-75 centile $0 \cdot 0-4 \cdot 0) \quad v$. 2.0 (25-75 centile $0 \cdot 0-7 \cdot 0)$, respectively; $P=0.01)^{(31)}$. Only studies investigating formulae supplemented with GOS/FOS reported a beneficial effect of prebiotic supplementation. Ivakhnenko \& Nyankovskyy ${ }^{(20)}$ found a significant reduction in the incidence of gastrointestinal tract infections at the age of 18 months in the GOS/FOS-supplemented $(0 \cdot 8 \mathrm{~g} / 100 \mathrm{ml})$ formula group compared with the unsupplemented formula group $(0 \cdot 28$ (SE 0.05) v. 0.78 (SE 0.12) episodes/ child/18 months, respectively; MD $-0.5 ; 95 \%$ CI $-0.53,-0.47$; $P<0.001)$. Bruzzese et $a{ }^{(18)}$ reported, in the GOS/FOSsupplemented formula group compared with the unsupplemented formula group, a reduced rate of diarrhoeal episodes (defined as 3 or more loose or watery stools/d lasting for at least $3 \mathrm{~d}$ ) (0.12 (sE 0.04) v. 0.29 (sE 0.05) episodes/child per 12 months, respectively; MD $-0 \cdot 17 ; 95 \% \mathrm{CI}-0 \cdot 18,-0 \cdot 16)$ and a reduced number of children with at least one episode of acute diarrhoea (10/96 v. 26/109, respectively; RR 0.37; $95 \%$ CI $0 \cdot 17,0 \cdot 82)$.

Respiratory tract infections. Only five studies reported the effects of prebiotic supplementation on the incidence of respiratory tract infections. No significant differences between groups in the number of episodes of upper respiratory tract infections per infant (1.65 (SE 1.8) v.1.84 (SE 2.0), respectively; $P=0.44$ ) or in the number of infants with at least three episodes of upper respiratory tract infections per year $(15.9 v \cdot 16.7 \% \text {, respectively; } P=0.9)^{(14)}$ were reported when GOS-supplemented formula and unsupplemented formula were compared. No significant differences between groups were reported in the median adjusted number of upper respiratory tract infections as a suspected cause of fever as well as the number of episodes or duration in days of coughing (episodes 2.55 (25-75 centile 1.09-4.07) v. 2.63 (25-75 centile 1.09-4.56), respectively, $P=038$; duration: 22.5 (25-75 centile 8.0-42.5) v. 24.0 (25-75 centile 9.0-50.0), respectively, $P=0 \cdot 27)$ or runny or blocked nose (episodes: 2.59 (25-75 centile 1.025.26) v. 2.91 (25-75 centile 0.99-4.95), respectively, $P=0.93$; duration: $21 \cdot 0$ (25-75 centile 5.0-61.0) v. 24.0 (25-75 centile 6.0-60.0), respectively, $P=0 \cdot 66$ ) between the prebiotics (FOS/ GOS) and control groups during 12 months and at 3-5 years ${ }^{(31,34)}$. Similar results were reported by Bruzzese et al. ${ }^{(18)}$. These authors found no significant differences between the FOS/GOS-supplemented formula group and the unsupplemented formula group in the number of patients with at least 1 episode of upper respiratory tract infections (60/94 v. 65/109, respectively; RR 1.07; 95\% CI
$0 \cdot 86,1.33)$ and $>3$ episodes of upper respiratory tract infections (17/60 v. 29.65, respectively; RR 0.64; $95 \%$ CI 0.39, 1.03). Also, Closa-Monasterolo et al. ${ }^{(28)}$ reported no significant differences in the mean number of infections between control and oligofructose-enriched inulin formula-fed groups. However, a significant reduction in the incidence of upper respiratory tract infections at the age of 18 months in the FOS/GOS-supplemented $(0 \cdot 8 \mathrm{~g} / 100 \mathrm{ml})$ formula group compared with the control formula group (2.81 (SE 0.51) v. 5.78 (SE 0.97), respectively; MD -2.97; $95 \%$ CI $-3 \cdot 26,-2 \cdot 68)$ was reported by Ivakhnenko \& Nyankovskyy ${ }^{(20)}$.

Allergic manifestations. Only two studies evaluated the effects of prebiotic supplementation on allergic manifestation. Sierra et $a l^{(14)}$ reported no significant difference between the unsupplemented and GOS-supplemented formula groups in the number of allergic manifestations (atopic dermatitis, wheezing, food allergy) (28/132 v. 39/172, respectively; RR 1.39; 95\% CI 0.91, $2 \cdot 12 ; \quad P=0 \cdot 12)^{(14)}$. Whereas Ivakhnenko \& Nyankovskyy ${ }^{(20)}$ found, in the GOS/FOS-supplemented formula group compared with the unsupplemented formula group, a significant reduction in the number of infants with allergic reactions to food (3/62 v. 9/53, respectively; RR 0.28; $95 \%$ CI 0.09, 0.9), allergic reactions to cows' milk protein $(2 / 62$ v. 8/53, respectively; RR $0 \cdot 2 ; 95 \% \mathrm{CI}$ $0.05,0.84)$, atopic dermatitis $(3 / 62 v .9 / 53$, respectively; RR 0.28 ; $95 \%$ CI $0.09,0 \cdot 9)$, and gastrointestinal symptoms of food allergy (2/62 v. 7/53, respectively; RR 0.24; 95\% CI 0.06, 0.98); however, there was no effect of formula supplementation on respiratory system allergic symptoms $(3 / 62 v .7 / 53$, respectively; RR $0 \cdot 36$; $95 \%$ CI $0 \cdot 1,1 \cdot 2$ ).

Antibiotic treatment. Five studies reported on the effects of prebiotic supplementation on frequency of antibiotic treatment. Four of them reported no significant difference between the unsupplemented and supplemented formula groups. Ripoll et $a l^{(13)}$ evaluated FOS-supplemented formula and reported no significant difference $(P=0 \cdot 12)$ in the number of infants with concomitant treatments (including antibiotics) between study groups. Sierra et $a l .{ }^{(14)}$ reported no significant difference between the unsupplemented and GOS-supplemented formula groups in the percentage of patients with antibiotic treatment (19.8 v. 17.8\%, respectively; RR $0.88 ; 95 \% \mathrm{CI} 0.5,1.45 ; P=0.48)$. In the studies by van Stuijvenberg et $a l .{ }^{(34)}$, there were no significant differences between the experimental (FOS/GOS-supplemented formula) and control groups in the median adjusted numbers of fever episodes for which systemic antibiotics were used during the 1st year of life as well as during follow-up of that group at $3-5$ years ${ }^{(31)}$. However, Bruzzese et al. ${ }^{(18)}$ found, in the FOS/GOS-supplemented formula group compared with unsupplemented formula group, a significantly reduced rate of antibiotic courses prescribed for children (1.03 (SE 0.15) v. 1.48 (SE 0.16), respectively; MD 0.45; $95 \%$ CI $-0.49,-0 \cdot 4, P=0 \cdot 038$ ) and a significantly reduced number of children receiving more than two antibiotic courses/year (24/60 $v$. 43/65, respectively; RR 0.6; 95\% CI 0.42, 0.86); however, there was only a borderline significant difference between groups in the number of children receiving at least 1 antibiotic course (46/60 v. 58/65, respectively; RR 0.86; $95 \%$ CI 0.73, 1.01).

Adverse events. A number of studies reported data on adverse events (Table 1), but none of them reported serious adverse events 
related to the tested formulae. Some authors defined adverse events as concomitant infections (nasopharyngitis, bronchitis, gastroenteritis and diarrhoea), whereas others defined adverse events as feeding-related gastrointestinal symptoms or symptoms of intolerance. No significant differences in the number of adverse events between supplemented and unsupplemented formula groups were reported by Ripoll $e t a l .{ }^{(13)}$ and Wernimont $e t a l .{ }^{(32)}$ evaluating FOS supplementation, by Piemontese et al. ${ }^{(24)}$ evaluating the FOS/GOS/ AOS supplementation, and by Scalabrin et al. ${ }^{(26)}$ evaluating PDX/ GOS supplementation. Ashley et al. ${ }^{(5)}$ evaluated GOS supplementation and PDX/GOS supplementation and found no differences in the overall incidence of at least one medically confirmed adverse event between supplemented and unsupplemented formula groups. However, these authors reported that the frequency of excessive spitting was significantly higher in the GOSsupplemented formula group $(7.5 v .0 \%, P<0.05)$ and gas was significantly less frequent in the PDX/GOS-supplemented group $(P<0.05)$ compared with the unsupplemented group. Also, Williams et $a l .{ }^{(15)}$ reported no significant differences among feeding groups in the proportions of subjects with specific adverse events, with one exception; there was a significantly higher proportion of serious adverse events, primarily respiratory tract infections, in the group that received a higher concentration of GOS $(0 \cdot 8 \mathrm{~g} / 100 \mathrm{ml} v$. $0.4 \mathrm{~g} / 100 \mathrm{ml} ; P<0.05)$. In two studies by Giovannini et $a l^{(11,39)}$, there were no differences in crying episodes, vomiting, or diarrhoeal episodes between the groups. However, the authors reported significantly lower regurgitation rates and episodes of infantile colic in the GOS-supplemented formula group $(P<0.05)$, and lower stool frequency in the unsupplemented formula group $(P<0.05)$. In a study evaluating oligofructose-enriched inulin supplementation, the frequencies of the majority of adverse events were generally equal in both groups; exceptions were the amount of time spent crying $(\mathrm{min} / \mathrm{d})$, which was significantly longer in the prebiotic group compared with the control group $(P<0.05)$, as well as the number of episodes of loose stools, which was slightly fewer in the prebiotic group compared with the control group (2 v. 8\%; $P<0.05)^{(28)}$. In some studies, no adverse events were observed throughout the study period in the control and experimental groups (GOS ${ }^{(33)}$, FOS/GOS) $)^{(16,18,22,23)}$.

\section{Non-clinical outcomes}

The online Supplementary Table S3 provides an overview of the non-clinical findings in all RCT that evaluated the effects of prebiotic-supplemented formulae. The findings were not consistent. However, differences differences in microbiota composition and immune parameters in infants fed prebioticsupplemented formula $v$. unsupplemented formula (e.g. higher stool colony counts of bifidobacteria; increased SCFA concentrations; reduce faecal $\mathrm{pH}$ ) were found.

\section{Discussion}

\section{Summary of findings}

We updated the 2011 evidence on the effects of the administration of prebiotic-supplemented infant formulae compared with unsupplemented formulae. Forty-one eligible trials were identified, described in twenty-five new publications.
Considering all of the evidence available (i.e. included in the earlier report by the Committee on Nutrition and the current up-date), supplementation of infant formula with FOS alone had no effect on growth, tolerance (assessed as some of following: abdominal pain, crying, nausea, vomiting, regurgitation), gastrointestinal infections, and stool frequency. However, it was associated with a softer stool consistency. Supplementation of infant formula with GOS alone had no effect on growth, tolerance (assessed as some of following: crying, regurgitation, fussiness, gassiness, vomiting, flatulence), gastrointestinal infections, respiratory tract infections, and allergic manifestations. However, it was associated with an increased stool frequency with a softer stool consistency in some trials. Supplementation of infant formula with FOS/GOS had no effect on growth or tolerance (assessed as some of following: crying, regurgitation, fussiness, gassiness, vomiting, flatulence, cramps). The effects on gastrointestinal and respiratory tract infections remain unclear, as this was documented in only some trials. Furthermore, the methodological quality of some them was poor. There was no effect of supplementation of infant formula with FOS/GOS on stool frequency. However, it was associated with a softer stool consistency. Supplementation of infant formula with AOS alone had no effect on growth, tolerance (assessed as crying, regurgitation, or vomiting), and stool frequency. However, it was associated with a softer stool consistency. Supplementation of infant formula with FOS/GOS/AOS had no effect on growth or tolerance (assessed as crying, regurgitation, vomiting). The effects on gastrointestinal and respiratory tract infections remain unclear, as this was documented in only some trials. Furthermore, the methodological quality of some them was poor. Supplementation of infant formula with FOS/GOS/AOS was associated with an increased stool frequency, with a softer/lower stool consistency. Supplementation of infant formula with PDX/GOS had no effect on growth or tolerance (as assessed by fussiness, gassiness). However, it was associated with a softer stool consistency. Supplementation of infant formula with oligofructose-enriched inulin had no effect on growth or tolerance (assessed as fussiness, regurgitation, or vomiting). However, it was associated with a softer stool consistency. Overall, adverse events were frequently reported; however, with minor exceptions, no differences were found between the prebiotic-supplemented and unsupplemented formula groups. None of the trials reported serious adverse events related to use of the study products.

Based on clinical outcomes, a number of studies analysed the effects of different prebiotics on growth, an essential outcome measure for evaluating the safety of infant formulae. All of these trials concluded that prebiotic supplementation of infant formulae did not have any significant effects on growth. All trials consistently showed that prebiotic supplementation of infant formulae has the potential to soften stools. However, the clinical significance of these findings remains unclear, although it may be beneficial in small infants with hard stools. Many, albeit not all, studies showed that prebiotic supplementation of infant formulae significantly increased stool frequency. Again, the clinical significance of these findings remains unclear. There is some inconsistent evidence suggesting that supplementation of infant formula with GOS/FOS may be associated with a reduced risk of 
gastrointestinal infections. However, the effect size was small and the confidence intervals were wide, so these results should be interpreted with caution. The supplementation of infant formula with GOS/FOS/AOS may be associated with a reduction in the duration of diarrhoea. With one exception, the available data showed no effect of prebiotic supplementation of infant formulae on respiratory tract infections. The only trial suggesting that supplementation of infant formula with GOS/FOS may be associated with a reduced risk of upper respiratory tract infections needs to be interpreted with caution, as the confidence intervals were wide. The effects of prebiotic supplementation of infant formulae on allergic diseases were inconclusive. One study with methodological limitations demonstrated that supplementation of infant formula with GOS/FOS resulted in a significant decrease in some allergic reactions. Supplementation with GOS/FOS/AOS reduced the risk of eczema. Supplementation with GOS had no effect on the rates of allergic manifestations and sensitisation. Thus, there is still too much uncertainty to draw reliable conclusions from the available data. The effects of prebiotic supplementation of infant formulae on antibiotic use were inconclusive. One study demonstrated that supplementation of infant formula with GOS/FOS resulted in a significant decrease in the number of fever episodes for which systemic antibiotics were used; the authors of other studies reported no significant decrease in antibiotic use with prebiotic supplementation. The reporting of symptoms such as crying, fussiness, regurgitation, and vomiting was not consistent. In trials in which these symptoms were evaluated as tolerance, no significant differences were found between the prebiotic-supplemented and unsupplemented formula groups. In trials in which these symptoms were evaluated as adverse events, differences were observed in some of the trials, but the effects were not consistent.

\section{Strengths and limitations}

An important strength of this systematic review is the use of rigorous methodology developed by the Cochrane Collaboration. The review addressed a clear question that was defined in terms of the study design, participants and interventions; however, the primary outcomes were not determined in advance.

We employed several methods to reduce bias (i.e. comprehensive literature search, pre-specified criteria for methodological assessment and analysis, no restrictions by language or year of publication). However, we cannot exclude the risk of publication bias, which was not formally addressed due to the limited number of included studies. The methodological quality of the included studies was generally moderate to low, which increases the risk of bias. Some of the prebiotics were evaluated in single trials only. The included studies were likely to be underpowered for addressing some outcomes (also adverse events).

The majority of included studies were industry-supported trials. There is bias associated with study funding sources. Compared with non-industry-sponsored studies, industrysponsored studies tended to have more favourable effectiveness and harm findings and more favourable conclusions ${ }^{(45)}$. Funding of research by manufacturers of infant formulae may be considered even more controversial because of the need for protection and promotion of breast-feeding. However, in the case of studies involving infant formulae, industry involvement is unavoidable, as investigators lack the means to manufacture quality infant products.

\section{Comparison with other studies}

Overall, even as new data have become available, the conclusions made previously did not change. With regard to growth, the 2011 systematic review by the ESPGHAN concluded that prebiotic-supplemented formulae do not raise safety concerns, that is, they do not have adverse effects on growth ${ }^{(1)}$. Our review does not confirm the findings of a 2012 systematic review by Mugambi et al., which concluded that prebioticsupplemented formula increased weight gain; however, currently, more data are available. Similar to our review, Mugambi et $a l .{ }^{(46)}$ concluded that use of prebiotic-supplemented formula increased stool frequency but, in contrast to our findings, it had no impact on stool consistency.

Our review found that all of the prebiotic-supplemented formulae were well tolerated; no serious adverse effects related to use of the study products were observed in any of the studies, regardless of the dose of prebiotics used. The dose is important. As recently concluded by Gibson et al. ${ }^{(47)}$, 'an appropriate dose must be sufficient to generate a prebiotic effect, but not too high to induce unwanted or adverse effects such as excessive gas formation or non-selective utilisation.'

Previously, the ESPGHAN Committee on Nutrition concluded that prebiotic supplementation of infant formulae has the potential to affect a number of non-clinical outcomes ${ }^{(1)}$. Overall, one of the most consistent findings was that the use of prebiotic-supplemented formulae resulted in significantly higher stool colony counts of bifidobacteria. It is generally accepted that the establishment of gut microbiota is of great importance to gastrointestinal physiology and appears to modulate the health and well-being of the host organism. The lower incidence of gastrointestinal and other infections found in breast-fed infants may, in part, be related to a predominance of Bifidobacterium and Lactobacillus in their gut microbiota. Therefore, the establishment of a gut microbiota closer to that of breast-fed infants in formula-fed infants after supplementation with prebiotics might be considered in the context of the current hypothesis on the role of the gut microbiota in health and disease. The development of not only infectious diseases but also non-communicable diseases (e.g. allergy, diabetes, obesity) may be related to aberrant gut microbiota early in life. At least in some studies, it has been documented that prebiotic supplementation stimulates the production of SCFA, primarily acetic acid. SCFAs are measurable products of bacterial fermentation and play a role in normal colonic functions. However, whether the increase in SCFA concentrations per se is of benefit is currently not well established.

The same applies to other stool parameters, such as the reduced faecal $\mathrm{pH}$ values in infants who have received prebiotic-supplemented formulae. The effects of prebioticsupplemented formulae on some immunologic parameters were not consistent. However, some effects may be potentially important. For example, prebiotic supplementation increased 
faecal IgA secretion. Considering that $\operatorname{IgA}$ is an antibody that plays a critical role in mucosal immunity, prebiotic supplementation may have an impact on the development of the immune system in infants. However, taken together, the interpretation of the non-clinical findings was difficult. Whether a change in any of these parameters per se is of benefit to the infants is currently not established.

\section{Conclusions}

In line with the 2011 ESPGHAN document, the available scientific data suggest that the administration of currently evaluated prebiotic-supplemented formulae to healthy infants does not raise safety concerns with regard to growth and adverse effects. Some favourable clinical effects are possible, primarily stool softening, which may be beneficial in some infants. Currently, there is no existing robust evidence to recommend the routine use of prebiotic-supplemented formulae. The latter conclusion may reflect the small amount of data on specific prebiotics and outcomes, rather than a genuine lack of an effect. The efficacy and safety should be considered for each prebiotic(s)-supplemented formula.

\section{Acknowledgements}

M. K. and M. P.-L. received travel funds to attend scientific meetings from companies manufacturing infant formulae. This study was funded in full by The Medical University of Warsaw.

All authors contributed to the initial protocol of the study. A. S., M. P.-L. and M. K. were responsible for data collection, data analysis and data interpretation. A. S. assumed the main responsibility for the writing of the first draft of the manuscript. All authors contributed to (and agreed upon) the final version.

H. S. had academic-associated speaking engagements and/or received research funding from companies manufacturing infant formulae. The remaining authors declare no conflicts of interest.

\section{Supplementary material}

For supplementary material/s referred to in this article, please visit https://doi.org/10.1017/S0007114518000120

\section{References}

1. Braegger C, Chmielewska A, Decsi T, et al. (2011) ESPGHAN Committee on Nutrition. Supplementation of infant formula with probiotics and/or prebiotics: a systematic review and comment by the ESPGHAN committee on nutrition. $J$ Pediatr Gastroenterol Nutr 52, 238-250.

2. Skórka A, Pieścik-Lech M, Kołodziej M, et al. (2017) To add or not to add probiotics to infant formulae? An updated systematic review. Benef Microbes 8, 717-725.

3. Higgins JPT \& Green S (editors) (2011) Cochrane Handbook for Systematic Reviews of Interventions Version 5.1.0 (updated March 2011). London: The Cochrane Collaboration. www. cochrane-handbook.org

4. Fanaro S, Jelinek J, Stahl B, et al. (2005) Acidic oligosaccharides from pectin hydrolysate as new component for infant formulae: effect on intestinal flora, stool characteristics, and pH. J Pediatr Gastroenterol Nutr 41, 186-190.
5. Ashley C, Johnston WH, Harris CL, et al. (2012) Growth and tolerance of infants fed formula supplemented with polydextrose (PDX) and/or galactooligosaccharides (GOS): double-blind, randomized, controlled trial. Nutr J 11, 38.

6. Ben XM, Zhou XY, Zhao WH, et al. (2004) Supplementation of milk formula with galacto-oligosaccharides improves intestinal micro-flora and fermentation in term infants. Chin Med $J$ (Engl) 117, 927-931.

7. Ben XM, Li J, Feng ZT, et al. (2008) Low level of galactooligosaccharide in infant formula stimulates growth of intestinal Bifidobacteria and Lactobacilli. World J Gastroenterol 14, 6564-6568.

8. Bettler J \& Euler AR (2006) An evaluation of the growth of term infants fed formula supplemented with fructooligosaccaride. Int J Probiotics Prebiotics 1, 19-26.

9. Brunser O, Figueroa G, Gotteland M, et al. (2006) Effects of probiotic or prebiotic supplemented milk formulas on fecal microbiota composition of infants. Asia Pac J Clin Nutr 15 , 368-376.

10. Fanaro S, Marten B, Bagna R, et al. (2009) Galactooligosaccharides are bifidogenic and safe at weaning: a double-blind randomized multicenter study. I Ped Gastroenterol Nutr 48, 82-88.

11. Giovannini M, Verduci E, Zuccotti G, et al. (2013) Safety of a formula supplemented with galactooligosaccharides in term infants. Int J Probiotics Prebiotics 8, 67-74.

12. Paineau D, Respondek F, Menet V, et al. (2014) Effects of short-chain fructooligosaccharides on faecal bifidobacteria and specific immune response in formula-fed term infants: a randomized, double-blind, placebo-controlled trial. J Nutri Sci Vitaminol 60, 167-175.

13. Ripoll C, Chappui E, Respondek F, et al. (2015) ScFOS supplemented follow-on formula in healthy infants: Impact on vaccin especific faecal secretory IGA response, faecal bifidobacteria, growth and digestive tolerance. Bioact Carbohydrates Diet Fibre 5, 169-178.

14. Sierra C, Bernal MJ, Blasco J, et al. (2015) Prebiotic effect during the first year of life in healthy infants fed formula containing GOS as the only prebiotic: a multicentre, randomised, doubleblind and placebo-controlled trial. Eur J Nutr 54, 89-99.

15. Williams $\mathrm{T}$, Choe $\mathrm{Y}$, price $\mathrm{P}$, et al. (2014) Tolerance of formulas containing prebiotics in healthy, term infants. J Pediatr Gastroenterol Nutr 59, 653-658.

16. Bisceglia M, Indrio F, Riezzo G, et al. (2009) The effect of prebiotics in the management of neonatal hyperbilirubinaemia. Acta Paediatr 98, 1579-1581.

17. Costalos C, Kapik A, Apostolou M, et al. (2008) The effect of a prebiotic supplemented formula on growth and stool microbiology of term infants. Early Hum Dev 84, 45-49.

18. Bruzzese E, Volpicelli M, Squeglia V, et al. (2009) A formula containing galacto- and fructo-oligosaccharides prevents intestinal and extra-intestinal infections: an observational study. Clin Nutr 28, 156-161.

19. Decsi T, Arató A, Balogh M, et al. (2005) Randomised placebo controlled double blind study on the effect of prebiotic oligosaccharides on intestinal flora in healthy infants. Orv Hetil 146, 2445-2450.

20. Ivakhnenko OS \& Nyankovskyy SL (2013) Effect of the specific infant formula mixture of oligosaccharides on local immunity and development of allergic and infectious disease in young children: randomized study. Pediatria Polska 88, 398-404.

21. Moro G, Minoli I, Mosca M, et al. (2002) Dosage-related bifidogenic effects of galacto- and fructooligosaccharides in formula-fed term infants. I Pediatr Gastroenterol Nutr 34, 291-295. 
22. Salvini F, Riva E, Salvatici E, et al. (2011) A specific prebiotic mixture added to starting infant formula has long-lasting bifidogenic effects. J Nutr 141, 1335-1339.

23. Veereman-Wauters $\mathrm{G}$, Staelens $\mathrm{S}$, Van de Broek $\mathrm{H}$, et al. (2011) Physiological and bifidogenic effects of prebiotic supplements in infant formulae. J Pediatr Gastroenterol Nutr 52, $763-771$.

24. Piemontese $\mathrm{P}$, Giannì ML, Braegger $\mathrm{CP}$, et al. (2011) Tolerance and safety evaluation in a large cohort of healthy infants fed an innovative prebiotic formula: a randomized controlled trial. PLOS ONE 6, e28010.

25. Nakamura N, Gaskins HR, Collier CT, et al. (2009) Molecular ecological analysis of fecal bacterial populations from term infants fed formula supplemented with selected blends of prebiotics. Appl Environ Microbiol $\mathbf{7 5}$ $1121-1128$.

26. Scalabrin DM, Mitmesser SH, Welling GW, et al. (2012) New prebiotic blend of polydextrose and galacto-oligosaccharides has a bifidogenic effect in young infants. $J$ Pediatr Gastroenterol Nutr 54, 343-352.

27. Ziegler E, Vanderhoof JA, Petschow B, et al. (2007) Term infants fed formula supplemented with selected blends of prebiotics grow normally and have soft stools similar to those reported for breast-fed infants. J Pediatr Gastroenterol Nutr 44, 359-364.

28. Closa-Monasterolo R, Gispert-Llaurado $\mathrm{M}$, Luque $\mathrm{V}$, et al. (2013) Safety and efficacy of inulin and oligofructose supplementation in infant formula: results from a randomized clinical trial. Clin Nutr 32, 918-927.

29. Xia Q, Williams T, Hustead D, et al. (2012) Quantitative analysis of intestinal bacterial populations from term infants fed formula supplemented with fructo-oligosaccharides. I Pediatr Gastroenterol Nutr 55, 314-320.

30. Knol J, Scholtens P, Kafka C, et al. (2005) Colon microflora in infants fed formula with galacto- and fructo-oligosaccharides: more like breast-fed infants. J Ped Gastroenterol Nutr 40, 36-42.

31. van Stuijvenberg M, Stam J, Grüber C, et al. (2015) Similar occurrence of febrile episodes reported in non-atopic children at three to five years of age after prebiotics supplemented infant formula. PLOS ONE 10, e0129927.

32. Wernimont $S$, Northington R, Kullen MJ, et al. (2015) Effect of an $\alpha$-lactalbumin-enriched infant formula supplemented with oligofructose on fecal microbiota, stool characteristics, and hydration status: a randomized, double-blind, controlled trial. Clin Pediatr (Phila) 54, 359-370.

33. Matsuki T, Tajima S, Hara T, et al. (2016) Infant formula with galacto-oligosaccharides (OM55N) stimulates the growth of indigenous bifidobacteria in healthy term infants. Benef Microbes 7, 453-461.

34. van Stuijvenberg M, Eisses AM, Grüber C, et al. (2011) Do prebiotics reduce the number of fever episodes in healthy children in their first year of life: a randomised controlled trial. Br J Nutr 106, 1740-1748.

35. Grüber C, van Stuijvenberg M, Mosca F, et al. (2010) Reduced occurrence of early atopic dermatitis because of immunoactive prebiotics among low-atopy-risk infants. J Allergy Clin Immunol 126, 791-797.

36. Scholtens PA, Alliet P, Raes M, et al. (2008) Fecal secretory immunoglobulin A is increased in healthy infants who receive a formula with short-chain galacto-oligosaccharides and longchain fructo-oligosaccharides. J Nutr 138, 1141-1147.

37. Bakker-Zierikzee AM, Alles MS, Knol J, et al. (2005) Effects of infant formula containing a mixture of galacto- and fructooligosaccharides or viable Bifidobacterium animalis on the intestinal microflora during the first 4 smonths of life. Br J Nutr 94, 783-790.

38. Bakker-Zierikzee AM, Tol EA, Kroes H, et al. (2006) Faecal SIgA secretion in infants fed on pre- or probiotic infant formula. Pediatr Allergy Immunol 17, 134-140.

39. Giovannini M, Verduci E, Gregori D, et al. (2014) Prebiotic effect of an infant formula supplemented with galactooligosaccharides: randomized multicenter trial. J Am Coll Nutri 33, 385-393

40. Alliet P, Scholtens P, Raes M, et al. (2007) Effect of prebiotic galacto-oligosaccharide, long-chain fructo-oligosaccharide infant formula on serum cholesterol and triacylglycerol levels. Nutrition 23, 719-723.

41. Salminen S, Endo A, Isolauri E, et al. (2016) Early gut colonization with lactobacilli and Staphylococcus in infants: the hygiene hypothesis extended. J Pediatr Gastroenterol Nutr 62, 80-86.

42. Haarman M \& Knol J (2006) Quantitative real-time PCR analysis of fecal Lactobacillus species in infants receiving a prebiotic infant formula. App Environ Microbiol 72, 2359-2365.

43. Raes M, Scholtens PA, Alliet P, et al. (2010) Exploration of basal immune parameters in healthy infants receiving an infant milk formula supplemented with prebiotics. Pediatr Allergy Immunol 21, e377-e385.

44. Stam J, van Stuijvenberg M, Garssen J, et al. (2011) A mixture of three prebiotics does not affect vaccine specific antibody responses in healthy term infants in the first year of life. Vaccine 29, 7766-7772.

45. Lundh A, Sismondo S, Lexchin J, et al. (2012) Industry sponsorship and research outcome. Cochrane Database Syst Rev, issue 12, MR000033.

46. Mugambi MN, Musekiwa A, Lombard M, et al. (2012) Synbiotics, probiotics or prebiotics in infant formula for full term infants: a systematic review. Nutr $J \mathbf{1 1}, 81$.

47. Gibson GR, Hutkins R, Sanders ME, et al. (2017) Expert consensus document: The International Scientific Association for Probiotics and Prebiotics (ISAPP) consensus statement on the definition and scope of prebiotics. Nat Rev Gastroenterol Hepatol 14, 491-502. 